\title{
The AKARI/IRC mid-infrared all-sky survey ${ }^{\star}$
}

D. Ishihara ${ }^{1,2}$, T. Onaka ${ }^{2}$, H. Kataza ${ }^{3}$, A. Salama ${ }^{4}$, C. Alfageme ${ }^{4, \star \star}$, A. Cassatella ${ }^{4,5,6}$, N. Cox ${ }^{4, \star \star \star}$, P. García-Lario ${ }^{4}$, C. Stephenson ${ }^{4, \dagger}$, M. Cohen ${ }^{7}$, N. Fujishiro ${ }^{3,8, \ddagger}$, H. Fujiwara ${ }^{2}$, S. Hasegawa ${ }^{3}$, Y. Ita ${ }^{9}$, W. Kim ${ }^{3,2, \S}$, H. Matsuhara ${ }^{3}$, H. Murakami ${ }^{3}$, T. G. Müller ${ }^{10}$, T. Nakagawa ${ }^{3}$, Y. Ohyama ${ }^{11}$, S. Oyabu ${ }^{3}$, J. Pyo ${ }^{12}$, I. Sakon ${ }^{2}$, H. Shibai ${ }^{13}$, S. Takita ${ }^{3}$,

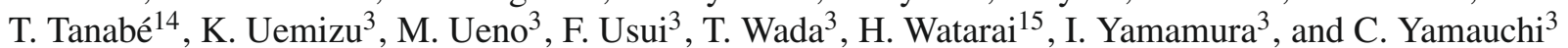

1 Department of Physics, Nagoya University, Furo-cho, Chikusa-ku, Nagoya, Aichi, 464-860, Japan e-mail: ishihara@u.phys.nagoya-u.ac.jp

2 Department of Astronomy, Graduate School of Science, University of Tokyo, 7-3-1 Hongo, Bunkyo-ku, Tokyo, 113-0033, Japan

3 Institute of Space and Astronautical Science (ISAS), Japan Aerospace Exploration Agency (JAXA), 3-1-1 Yoshinodai, Sagamihara, Kanagawa, 229-8510, Japan

4 European Space Astronomy Center (ESAC), Villanueva de la Cañada, PO Box 78, 28691 Madrid, Spain

5 INAF, Istituto di Fisica dello Spazio Interplanetario, via del Fosso del Cavaliere 100, 00133 Roma, Italy

6 Dipartimento di Fisica, Universita' Roma Tre, via della Vasca Navale 100, 00146 Roma, Italy

7 Radio Astronomy Laboratory, University of California, Berkeley, USA

8 Department of Physics, Faculty of Science, University of Tokyo, 3-1-1 Hongo, Bunkyo-ku, Tokyo, 113-0003, Japan

9 National Astronomical Observatory of Japan, Mitaka, Tokyo, 181-8588, Japan

${ }_{10}$ Max-Planck-Institut für extraterrestrische Physik, Giessenbachstraße, 85748 Garching, Germany

11 Academia Sinica, Institute of Astronomy and Astrophysics (ASIAA), Taipei 10617, Taiwan

12 Korea Astronomy and Space Science Institute (KASI), 61-1, Hwaam-dong, Yuseong-gu, Daejeon, 305-348, Republic of Korea

13 Graduate School of Science, Osaka University, 1-1, Machikaneyama, Toyonaka, Osaka, 560-0043, Japan

14 Institute of Astronomy, Faculty of Science, University of Tokyo, Mitaka, Tokyo, 181-8588, Japan

15 Space Applications Mission Directorate, Japan Aerospace Exploration Agency (JAXA), 2-1-1, Sengen, Tsukuba, Ibaraki, 305-8505, Japan

Received 6 December 2009 / Accepted 17 February 2010

\section{ABSTRACT}

Context. AKARI is the first Japanese astronomical satellite dedicated to infrared astronomy. One of the main purposes of AKARI is the all-sky survey performed with six infrared bands between $9 \mu \mathrm{m}$ and $200 \mu \mathrm{m}$ during the period from 2006 May 6 to 2007 August 28 . In this paper, we present the mid-infrared part ( $9 \mu \mathrm{m}$ and $18 \mu \mathrm{m}$ bands) of the survey carried out with one of the on-board instruments, the infrared camera (IRC).

Aims. We present unprecedented observational results of the $9 \mu \mathrm{m}$ and $18 \mu \mathrm{m}$ AKARI all-sky survey and detail the operation and data processing leading to the point source detection and measurements.

Methods. The raw data are processed to produce small images for every scan, and the point sources candidates are derived above the $5 \sigma$ noise level per single scan. The celestial coordinates and fluxes of the events are determined statistically and the reliability of their detections is secured through multiple detections of the same source within milli-seconds, hours, and months from each other. Results. The sky coverage is more than $90 \%$ for both bands. A total of 877091 sources $(851189$ for $9 \mu \mathrm{m}, 195893$ for $18 \mu \mathrm{m})$ are confirmed and included in the current release of the point source catalog. The detection limit for point sources is $50 \mathrm{mJy}$ and $90 \mathrm{mJy}$ for the $9 \mu \mathrm{m}$ and $18 \mu \mathrm{m}$ bands, respectively. The position accuracy is estimated to be better than $2^{\prime \prime}$. Uncertainties in the in-flight absolute flux calibration are estimated to be $3 \%$ for the $9 \mu \mathrm{m}$ band and $4 \%$ for the $18 \mu \mathrm{m}$ band. The coordinates and fluxes of detected sources in this survey are also compared with those of the IRAS survey and are found to be statistically consistent.

Key words. infrared: general - techniques: image processing - surveys

* Catalog is available in electronic form at the CDS via anonymous ftp to cdsarc.u-strasbg.fr $(130.79 .128 .5)$ or via

http://cdsweb.u-strasbg.fr/cgi-bin/qcat?J/A+A/514/A1

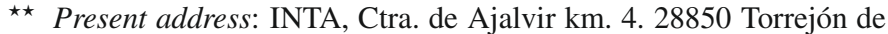
Ardoz, Madrid, Spain.

$\star \star \star$ Present address: Instituut voor Sterrenkunde, Katholieke Universiteit Leuven, Celestijnenlaan 200D, 3001 Leuven, Belgium.

Present address: Deimos Space S.L., Ronda de Poniente, 19, Edificio Fiteni VI, 28760 Tres Cantos, Madrid, Spain.

$¥$ Present address: Cybernet system Co. Ltd., 3 Kanda-neribeicho, Chiyoda-ku, Tokyo, 101-0022, Japan.

$\$$ Present address: SONY Co. Ltd., 4-14-1, Asahi-cho, Atsugi-shi, Kanagawa, 243-0014, Japan.

\section{Introduction}

Unbiased and sensitive all-sky surveys at infrared wavelengths are important for the various fields of astronomy. The first extensive survey of the mid- to far-infrared sky was made by the IRAS mission launched in 1983 (Neugebauer et al. 1984). IRAS surveyed $87 \%$ of the sky in four photometric bands at $12 \mu \mathrm{m}, 25 \mu \mathrm{m}$, $60 \mu \mathrm{m}$ and $100 \mu \mathrm{m}$ and substantially pioneered the various new fields of astronomy, like circumstellar debris disks around Vegalike stars (Aumann et al. 1984) and a new class of galaxies that radiate most of their energy in the infrared (Soifer et al. 1987). 
A decade later than IRAS, the Midcourse Space Experiment (MSX; Price et al. 2001) surveyed the Galactic plane as well as the regions not observed by or confused in the IRAS mission with higher sensitivity and higher spatial resolution $\left(18.3^{\prime \prime}\right)$ in four mid-infrared broad bands centered at $8.28 \mu \mathrm{m}, 12.13 \mu \mathrm{m}$, $14.65 \mu \mathrm{m}$ and $21.23 \mu \mathrm{m}$ and two narrow bands at $4.29 \mu \mathrm{m}$ and $4.35 \mu \mathrm{m}$. The MSX catalog (version 1.2) of the Galactic plane survey contains 323052 sources, three times as many as IRAS listed for the same region.

AKARI, the first Japanese space mission dedicated to infrared astronomical observations (Murakami et al. 2007), was launched in 2006 and was brought into a sun-synchronous polar orbit at an altitude of $700 \mathrm{~km}$. It has two scientific instruments, the infrared camera (IRC; Onaka et al. 2007) for 2-26 $\mu \mathrm{m}$ and the Far-Infrared Surveyor (FIS; Kawada et al. 2007) for 50-200 $\mu \mathrm{m}$. AKARI has a Ritchey-Chretien type cooled telescope with a primary-mirror aperture size of $685 \mathrm{~mm}$ (Kaneda et al. 2007), which is operated at $6 \mathrm{~K}$ by liquid helium and mechanical coolers. One of the major observational objectives of AKARI is an all-sky survey observation. The survey was executed during the life time of the cooling medium between 2006 May 8 and 2007 August 28. The $9 \mu \mathrm{m}$ and $18 \mu \mathrm{m}$ bands of the IRC and the $65 \mu \mathrm{m}, 90 \mu \mathrm{m}, 140 \mu \mathrm{m}$, and $160 \mu \mathrm{m}$ bands of the FIS were used for the all-sky survey.

In this paper, we present the mid-infrared part of the allsky survey performed with the IRC. The IRC was originally designed for imaging and spectroscopic observations in the pointing mode, but the all-sky observation mode was added as an operation mode following ground tests, in which the acceptable performance of continuous survey-type observations was confirmed (Ishihara et al. 2006a). The data of the IRC all-sky survey observation have been processed by a dedicated program and a point source catalog has been prepared. The content of this paper is based on the $\beta$ - 1 version of the AKARI/IRC all-sky survey point source catalog.

The outline of the observation is presented in Sect. 2. The data reduction is described in Sect. 3. The quality of the cata$\log$ is statistically evaluated in Sect. 4, and a summary is given in Sect. 5.

\section{Observations}

\subsection{The AKARI satellite}

The AKARI satellite has two observational modes: the all-sky survey and pointed observations. In the pointed observations, the telescope stares at the target or makes round trip scans around the target for about $10 \mathrm{~min}$. In the all-sky survey, the spacecraft spins around the Sun-pointed axis once every orbit, keeping the telescope toward a great circle and making continuous scans of the sky at a scan rate of $216^{\prime \prime} \mathrm{s}^{-1}$. The orbit rotates around the axis of the Earth at the rate of the yearly round of the earth. Thus, the whole sky is in principle covered in half a year.

In the first half year (Phase 1) of the mission the all-sky survey was dedicated as a first priority with pointed observations toward the North Ecliptic Pole and the Large Magellanic Cloud. In the second and third half year (Phase $2 \mathrm{a}$ and $2 \mathrm{~b}$ ), the all-sky survey was continued until the cooling medium (liquid helium) was exhausted. The time was divided between pointed observations survey observations to increase the final sky coverage of the all-sky survey.
Table 1. Parameters for mid-infrared all-sky survey operation.

\begin{tabular}{lcc}
\hline \hline Filter band (Camera) & $S 9 W($ MIR-S) & $L 18 W($ MIR-L) \\
\hline Wavelength & $6.7-11.6 \mu \mathrm{m}$ & $13.9-25.6 \mu \mathrm{m}$ \\
Isophotal wavelength & $8.61 \mu \mathrm{m}$ & $18.39 \mu \mathrm{m}$ \\
Effective bandwidth & $4.10 \mu \mathrm{m}$ & $9.97 \mu \mathrm{m}$ \\
Sampling rate (period) & $22.27 \mathrm{~Hz}(44 \mathrm{~ms})$ \\
Scan rate (exposure* $)$ & $216^{\prime \prime} \mathrm{s}^{-1}(11 \mathrm{~ms})$ \\
Reset rate (period) & \multicolumn{2}{c}{$0.074 \mathrm{~Hz}(13.464 \mathrm{~s})$} \\
Operation & \multicolumn{2}{c}{$256 \times 2$ pix } \\
Operated row & \multicolumn{2}{c}{$117^{\text {th }}, 125^{\text {th }}$} \\
Binning & $4 \times 1$ pix \\
Virtual pixel scale & $9 ! 36 \times 9.36$ & $10.4 \times 99^{\prime} .36$ \\
Detection limit $(5 \sigma)$ & $50 \mathrm{mJy}^{\dagger}$ & $120 \mathrm{mJy}^{\dagger}$ \\
\hline
\end{tabular}

Notes. ${ }^{(*)}$ Effective exposures for point sources are determined not by the sampling rate, but by the dwelling time of a source on a pixel.

(†) Estimated value from readout noise in shuttered configuration in-orbit.

(\$) Defined as where the responsivity for a given energy is larger than $1 / e$ of the peak.

\subsection{The infrared camera (IRC)}

The mid-infrared component of the AKARI all-sky survey was performed with one of the two focal-plane instruments: the IRC. The IRC covers the wavelength range of $2-26 \mu \mathrm{m}$ with three independent channels: NIR $(2-5.5 \mu \mathrm{m})$, MIR-S $(6-12 \mu \mathrm{m})$ and MIR-L (12-26 $\mu \mathrm{m})$. The IRC was primarily designed for deep imaging and spectroscopy in pointed observations. All the channels have filter wheels, which hold three filters and two spectroscopic dispersers. Each channel has a large format array that provides a wide field-of-view (FOV) of $10^{\prime} \times 10^{\prime}$. The MIR-S and MIR-L channels have infrared sensor arrays of $256 \times 256$ pixels (Si:As/CRC-744 manufactured by Raytheon). The pixel scales for MIR-S and MIR-L are 2'.34 × 2'.34 and 2'.51 × 2'.39, respectively. The field-of-views of MIR-S and MIR-L are separated by $20^{\prime}$ in the cross-scan direction. More details and the in-flight performance are described in Onaka et al. (2007).

\subsection{All-sky survey operations of the IRC}

Array operation. During the all-sky survey observations, only two out of 256 rows in the sensor array are operated in the continuous and non destructive readout mode (scan operation of the array; Ishihara et al. 2006a). The first row used in the operation can be selected arbitrarily out of 256 rows, and we adopted the 117 th row (hereafter row\#1). The second row (hereafter row\#2) was fixed to be eight rows from the first row the sampling rate of the array and the designed scan rate of the satellite.

The sampling rate was set to $22.72 \mathrm{~Hz}$ (one sampling per $44 \mathrm{~ms}$ ) taking account of the array operation conditions and the data down-link capacity. All the pixels were reset at a rate of $0.074 \mathrm{~Hz}$ (one reset per 306 samplings) to discharge the photo-current.

The NIR channel is not used during the all-sky survey because of the capacity of the down link rate and because the alignment of the NIR array is not suited for the all-sky survey observation.

Exposure time. The scan speed of the satellite in the survey observation mode is $216^{\prime \prime} \mathrm{s}^{-1}$. As the pixel scale of the detector in the in-scan direction is about 2 '. 34 , the resulting effective exposure time for a point source is $11 \mathrm{~ms}$. 


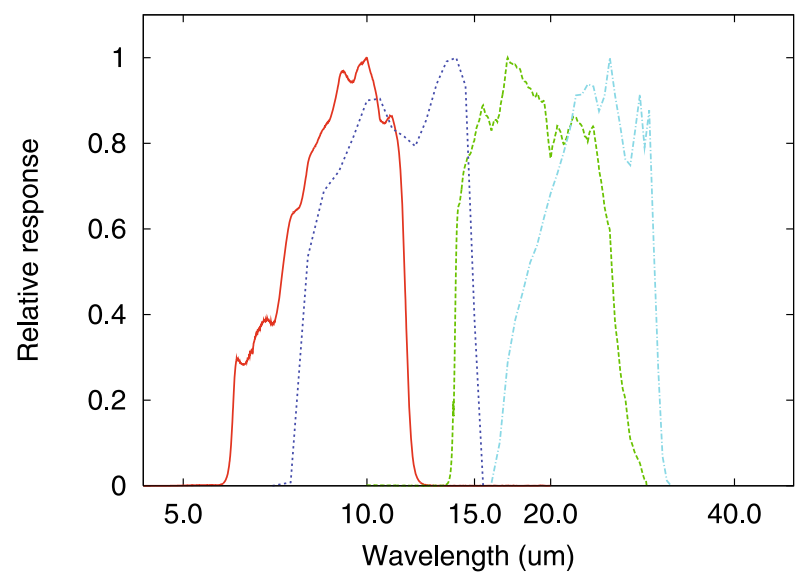

Fig. 1. Relative spectral response curve of the $S 9 W$ (solid curve) and $L 18 \mathrm{~W}$ bands (dashed) in units of electron/energy normalized to the peak. The system response curves of the IRAS $12 \mu \mathrm{m}$ (dotted) and $25 \mu \mathrm{m}$ bands (dashed-dotted) are also shown for comparison. The RSRs of the IRC are available at http://www.ir.isas. jaxa. jp/ASTRO-F/Observation/RSRF/IRC_FAD/index.html. The system response curves of the IRAS are taken from Table II.C.5 of IRAS Explanatory Supplement (Beichman et al. 1988).

Filter bands. The all-sky survey is performed with two broad bands centered at $9 \mu \mathrm{m}$ ( $S 9 \mathrm{~W}$ filter of MIR-S) and $18 \mu \mathrm{m}$ ( $L 18 W$ filter of MIR-L). The relative spectral response (RSR) curves of the two bands are shown in Fig. 1 together with those of the IRAS 12 and $25 \mu \mathrm{m}$ bands.

Pixel scale. The effective pixel size in the survey observation (hereafter virtual pixel size) is 9.'36 $\times 9$ '.36 for the $9 \mu \mathrm{m}$ band, and $10 .{ }^{\prime} 4 \times 9.36$ for the $18 \mu \mathrm{m}$ band. The in-scan pixel size is fixed by the sampling rate, while the outputs of four neighboring pixels are coadded to meet the down-link rate requirements and still make effective observations. A finer resolution is obtained in the data processing by combining images produced by the two rows. A detailed description of the reconstruction of the image and the resulting spatial resolution are given in Appendix A.

Confirmation strategy. It is difficult to distinguish events of real celestial objects from those due to cosmic ray hits only from the shape of signal because the virtual pixel size is not small enough to sample the PSF. We improved the reliability of source detections by adopting a three-step confirmation scheme.

The detection of a celestial source in row\#1 is confirmed by a second detection in row\#2 $87 \mathrm{~ms}$ later. In this process, a large fraction of the signals due to cosmic ray hits are expected to be removed (milli-seconds confirmation).

The width of the array in the cross-scan direction is about $10^{\prime}$. The scan path shifts at most by $4^{\prime}$ (on the ecliptic plane) due to the orbital motion. In this way the scan path overlaps at least by $6^{\prime}$ with the next scan 100 min after. The detection is thus confirmed by the next scan observation (hours confirmation).

Furthermore, the scan path rotates by 180 degrees around the axis of the Earth in half a year, giving another chance of detection six months later (months confirmation). Objects such as asteroids, comets and geostationary satellites, can be distinguished from stars and galaxies by hours and/or months-confirmations.

Sky coverage. Large portions of the sky have a chance to be covered more than three times during the survey period (Phase1,

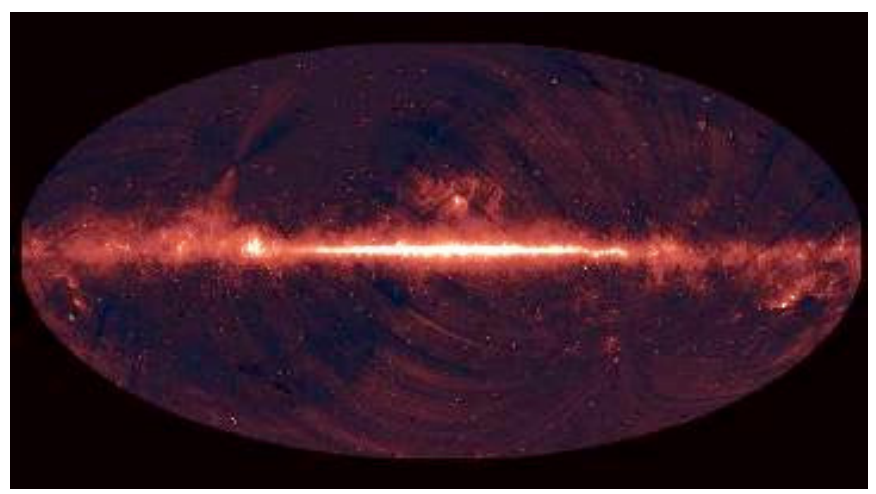

Fig. 2. All-sky image taken with the AKARI $9 \mu \mathrm{m}$ band, from which the zodiacal light is subtracted simply as the low spatial frequency component derived from the raw data.

Phase2a, and Phase2b; Sect. 2.1). During the semi-continuous survey, observational gaps (where no data are effectively available) appear for several reasons: (1) the survey observations are halted for every pointed observation. A sky area of about $10^{\prime} \times$ $120^{\circ}$ is skipped during a pointed observation. (2) During Phase 2 the attitude of the satellite was operated actively also in the allsky survey mode to cover the sky area not observed in Phase 1 in order to complete the far-infrared FIS all-sky survey (offset survey). But it is the intermission of the continuous survey for the mid-infrared channels that has FOV directions different from the FIS. (3) The mid-infrared survey observations are sometimes halted because of the limited downlink capacity. (4) The attitude of the satellite is lost due to a star-tracking failure. (5) The shutter is closed in the moon-avoidance region. (6) The data taken during resets and during times affected by the heavy reset anomaly are masked by the pipeline software (Sect. 3.1). (7) Saturated pixels cannot observe the sky until they have been reset (saturation trail).

Taking into account reasons (1), (2), and (3), which are automatically detected from the telemetry data, the final sky coverage is higher than $90 \%$ for both bands. Note that the actual sky area in which the source confirmation is carried out is smaller than this number (see Sect. 3.5). Figure 2 shows the AKARI $9 \mu \mathrm{m}$ low-resolution intensity map derived through the data processing described in this paper, from which the zodiacal light has been subtracted.

\section{Data processing}

The outline of the data processing (Fig. 3) for deriving the first point source catalog of the AKARI/IRC mid-infrared all-sky survey is summarized below.

- The raw telemetry data, which include the output of the sensor array, the house keeping data, and the output of the attitude control system, are down-linked from the satellite. Their timing is matched with each other and then they are registered to the database.

- The raw data that are sandwiched by two successive resets in the scan are processed to make pieces of images corresponding to $10^{\prime} \times 50^{\prime}$ wide sky regions (hereafter unit images) (basic process; Sect. 3.1).

- Then signals of the point source detections (events) are extracted from each processed image (event detection and milli-seconds confirmation; Sect. 3.2).

- Next, the coordinates of all the events are determined according to the output of the attitude control system and are 


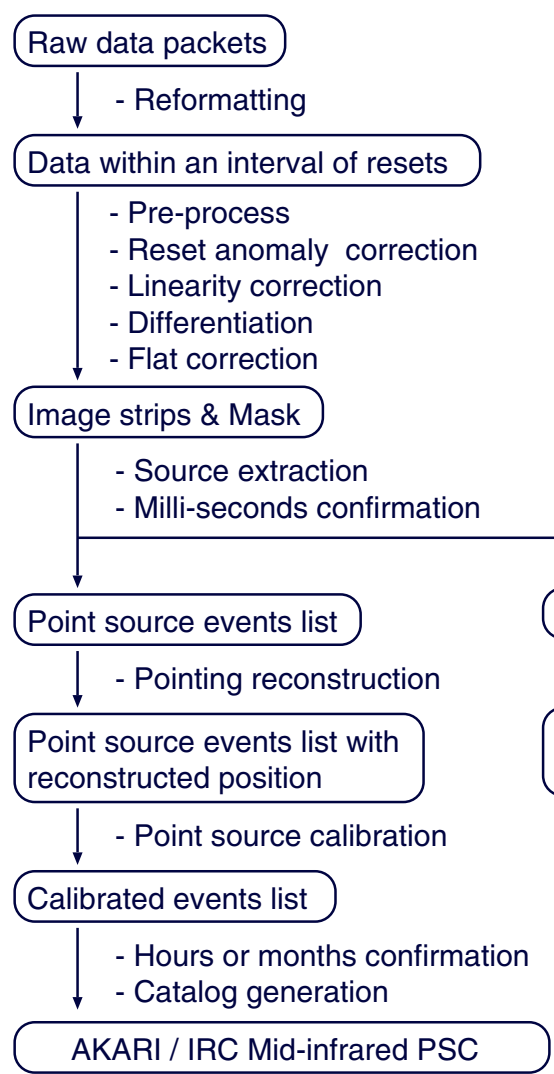

Fig. 3. Outline of AKARI/IRC all-sky survey data processing.

refined with the cross-correlations between the detected events and the prepared standard stars for the position determination (pointing reconstruction; Sect. 3.3).

- After the absolute flux calibration based on the measurements of standard stars, the fluxes of all the detected events are statistically derived (flux calibration; Sect. 3.4).

- Finally, reliable events are compiled into point source lists and the source lists of the $9 \mu \mathrm{m}$ band and $18 \mu \mathrm{m}$ band are merged to produce the IRC all-sky survey catalog (catalog compilation; Sect. 3.5).

\subsection{Basic process}

The basic process derives a unit image of $10^{\prime} \times 50^{\prime}$ from the raw data for the extraction of point source candidates.

ADU to electron conversion. First, the pixel value of the raw data is converted from ADU into electrons as

$S^{(1)}(i, t)=C F \cdot S^{(0)}(i, t)$,

where $i$ is the pixel number, $t$ is the time from the latest reset, $S^{(n)}(i, t)$ is the pixel value at $(i, t)$ as the result of applying the $n$th step, and $C F$ is the conversion factor measured from laboratory tests and assumed to be a constant for the pixels in the detector array (Ishihara et al. 2003).

Reset anomaly correction. An anomalous behavior of the output level that persists a few seconds after the reset (reset anomaly) is corrected. An example of this phenomenon and its correction are shown in Fig. 4. The offset level of the output of

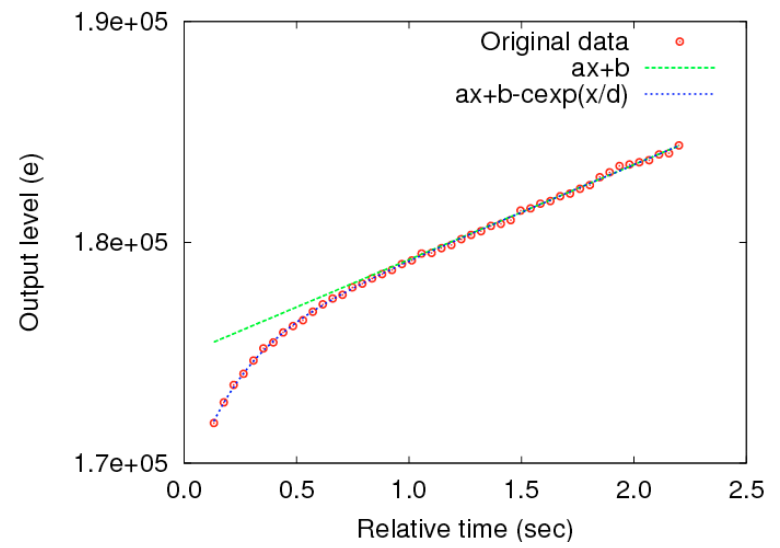

Fig. 4. Example of the reset anomaly correction. The output signal level of a pixel in units of electron is plotted against the time from latest reset. This data set was taken under the constant illuminating source in the laboratory test. The raw output of the sensor (solid points) is fitted by the reset anomaly function $O\left(t, e_{\text {int }}\right)$ (dotted curve) and corrected (dashed line)

the detector is fairly sensitive to the temperature (Ishihara et al. 2003). We thus suppose that this phenomenon is explained by the drift of the offset level of the read-out circuit, which is hybridized to the detector array because of the temperature drift invoked by the reset current to discharge the stacked photo-electrons. Assuming that this behavior is pixel-independent in the detector array, the reset anomaly is corrected as

$S^{(2)}(i, t)=S^{(1)}(i, t)-O\left(t, e_{\mathrm{res}}\right)$

where $i$ is the pixel number, $t$ is the time from latest reset, $e_{\text {res }}$ is (pixel average of) the amount of the photo-electrons discharged during the latest reset, and $O\left(t, e_{\text {res }}\right)$ is the offset level variation. The offset level variation $O\left(t, e_{\text {res }}\right)$ due to the reset is represented in the form of

$O\left(t, e_{\text {int }}\right)=C_{1} \exp \left(-t / C_{2}\right)$

where $C_{1}$ and $C_{2}$ are constants statistically derived from laboratory tests.

Masking invalid data A masking pattern is created to ignore unusable data. (1) All the pixels are masked during the resets and the periods heavily affected by the reset anomaly. (2) Saturated pixels are masked from the moment of the saturation to the next reset. (3) The pixels under the slit masks are always masked. Both MIR-S and MIR-L channels have a slit and slit masks at the edge of the FOV for spectroscopic observations of extended objects. The slit mask covers a few pixels located near the edge of the rows used in the survey operation.

Linearity correction. We corrected for the non linearity of the photo-response $\left(L\left(e_{\text {int }}\right)\right)$, which is supposedly due to the decrease in the bias voltage imposed on the detector array by the accumulation of the photo-electrons in each pixel. It is supposed to be a function of the number of stacked electrons $\left(e_{\text {int }}\right)$. An example of the non linearity is shown in Fig. 5. The non linearity of the photo-response is thus corrected as

$S^{(3)}(i, t)=S^{(2)}(i, t) / L\left(e_{\text {int }}\right)$ 


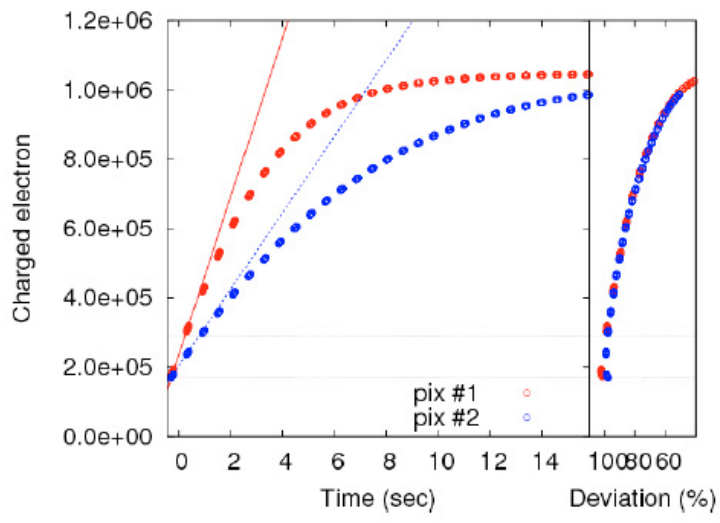

Fig. 5. Example of the non-linearity of the photo-response of a pixel. The left panel shows the time from reset versus output level of two pixels at the constantly illuminated condition in a laboratory test. The output profiles are fitted by linear functions using the data in the range, where the photo-response is still linear because the number of stacked electrons is small (7000-15000 ADU). The right panel shows the number of charged electrons ( $Y$-axis) versus the deviation of the output level from the linear functions ( $X$-axis).

assuming that their behaviors are the same for all the pixels. The non linearity function $L\left(e_{\text {int }}\right)$ is approximated by a spline function:

$L_{j}\left(e_{\text {int }}\right)=\sum_{i=0}^{3} C_{j}^{(i)} e_{\text {int }}^{i}$,

where $j(j=0,1,2)$ represents the $j$ th range of $e_{\text {int }}$ and each coefficient $\left(C_{j}^{(i)}\right)$ is derived from laboratory tests.

Differentiation. The signal is differentiated with respect to time,

$S^{(4)}(i, t)=S^{(3)}(i, t+\Delta t)-S^{(3)}(i, t)$,

where $\Delta t$ is the time step (44 ms) corresponding to the sampling rate.

Flat fielding. The differentiated signal $S^{(4)}(i, t)$ is corrected for flat-fielding.

$S^{(5)}(i, t)=S^{(4)}(i, t) / F(i)$,

where $F(i)$ is the normalized flat correction factor for each pixel operated in the survey mode representing the dispersion of the photo-response among the pixels. The flat function $F(i)$ is derived from multiple detections of the stars on different pixels. The background sky data are not used to avoid the effects of the scattered light. Figure 6 shows the flat functions for both rows in both bands.

Image construction from data of the two rows. The data from row\#1 and row\#2 are combined into a single image with a pixel scale of 1 '.56 to reject cosmic ray hits and produce a finer and higher $S / N$ image. Details on the combining process are given in Appendix A. The mask patterns from the two rows are also combined in the same manner. Figure 7 shows examples of the raw data obtained by the two rows, the processed data of the two rows, (i.e. the resulting combined image of $10^{\prime} \times 50^{\prime}$ ), and the combined mask pattern. The images and mask patterns are stacked as the basic calibrated data and put into the next step, the source extraction process.

\subsection{Event detection and milli-seconds confirmation}

After the basic processing, signals above $5 \sigma$ per scan are extracted from each image and checked by milli-seconds confirmation for point source detection (hereafter event). An example of these processes is shown in Fig. 8.

First, events are extracted from two images obtained independently by the two rows with the Source Extractor (Bertin et al. 2000) with a $3 \sigma$ threshold. Then events above $3 \sigma$ are extracted again on the finer combined image. Events from row\#1, row\#2 and the combined image are cross-identified by the detected position. Celestial sources are expected to be detected in both rows on the same pixel number $(i)$ with similar fluxes, whereas false detections like cosmic ray hits are expected to be detected only on one side. A pair of detections at the same sky position with similar fluxes $\left(0.1<F_{\text {row\#1 }} / F_{\text {row\#2 }}<10\right)$ are selected as milli-seconds confirmed sources. Finally, the flux (photometric result) and the position (timing and pixel number of the detection) of the combined image are recorded for the confirmed events.

\subsection{Pointing reconstruction}

The celestial coordinate of a source $\left(Q_{\mathrm{pix}}(i, t)\right)$ detected on the $i$ th pixel at the moment $t$ are derived from

$Q_{\mathrm{obj}}(i, t)=L(t) E(i) Q_{\mathrm{B}}(t)$,

where $Q_{\mathrm{B}}(t)$ is the boresight position of the telescope at the moment $t, E(i)$ is the conversion from the boresight to the $i$ th pixel, and $L(t)$ is the correction for the aberration due to the yearly revolution of the Earth and the orbital motion of the satellite.

The improvement of boresight position of the telescope $\left(Q_{\mathrm{B}}(t)\right)$ is carried out in collaboration with ESA by associating detected events in the $9 \mu \mathrm{m}$ and $18 \mu \mathrm{m}$ bands and signals of the focal-star sensors at near-infrared on the focal plane with the stars in the positional reference catalog prepared from MSX, 2MASS and IRAS (pointing reconstruction). The actual alignment of the FOV of each pixel $E(i)$ including the effect of the distortion is optimized statistically in the pointing reconstruction process.

Figure 9 shows the accuracy of the pointing reconstruction. The plot shows the fraction of the IRC events with an error smaller than the given values. The error is estimated from the distance between the positions determined from the pointing reconstruction and those from the position reference catalog. To make a fair evaluation, the pointing reconstruction for this test is carried out using randomly selected sources amounting to half of the catalog, and then the positions of the sources from the other half of the catalog are determined for the evaluation. The error includes the pointing reconstruction processing and the measurement errors. For the brightest sources the measurement error should be a minor contributor. We conclude that the position accuracy is better than $3^{\prime \prime}$ for $95 \%$ of the events.

Details of the pointing reconstruction are summarized in Salama et al. (2010, in prep.). We adopt the position of each detected event derived from the pointing reconstruction process.

\subsection{Flux calibration}

The photometric output for all the milli-seconds confirmed events is converted from electrons into physical units (Jy). The conversion function from electrons to Jy is derived statistically by comparing the model fluxes with the measurements of hundreds of standard stars. The standard stars are selected from 

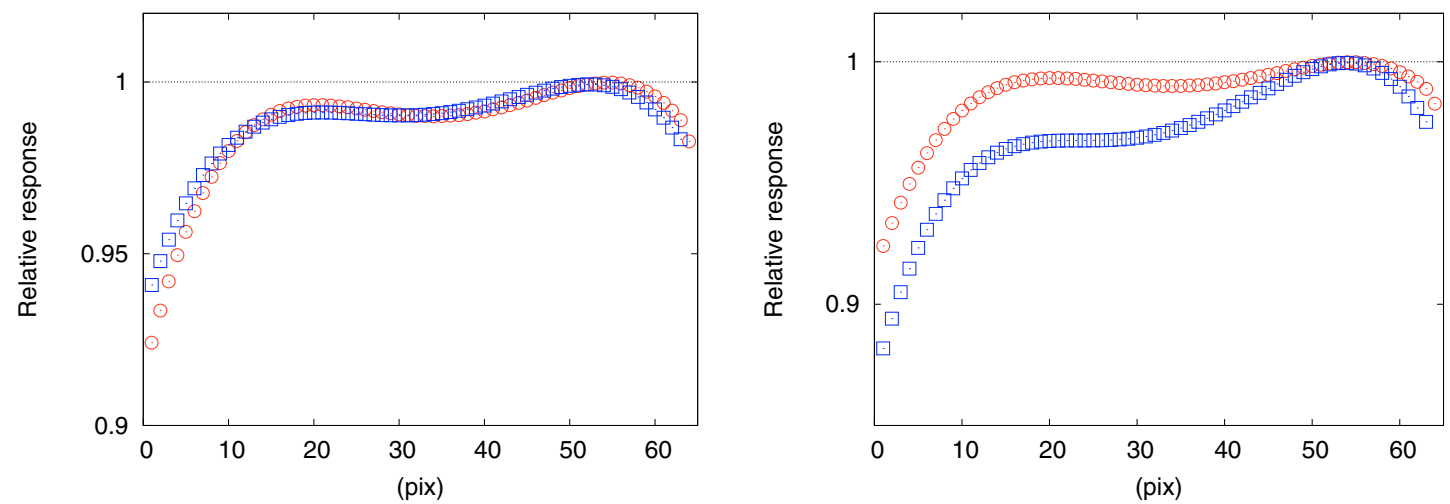

Fig. 6. Flat functions for the one dimensional array for the $9 \mu \mathrm{m}$ band (left) and $18 \mu \mathrm{m}$ band (right). The open circles represent row\#1 and open squares represent row\#2 in both panels.
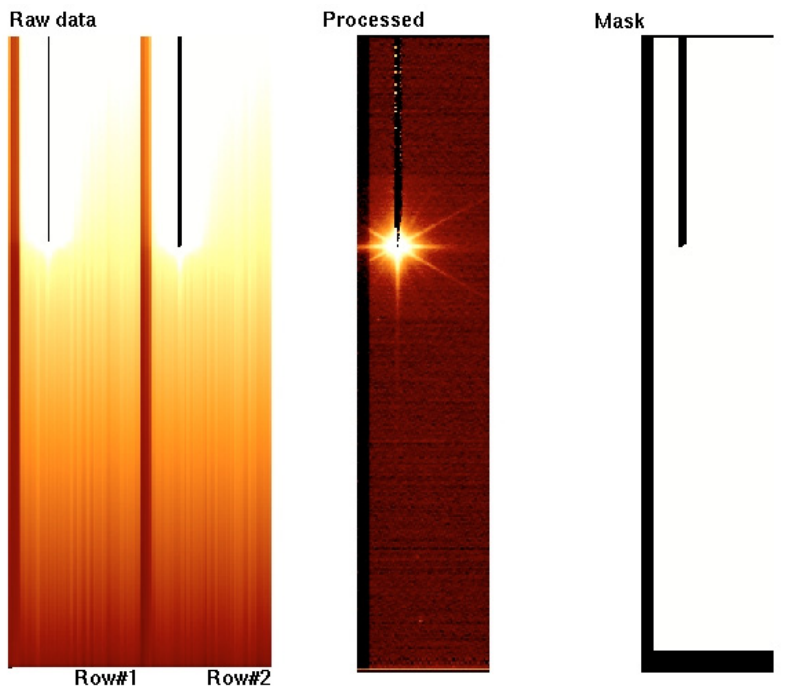

Fig. 7. Example of the processed data. (Left) Raw data from Row\#1 and Row\#2 are shown separately. (Middle) Processed data. The data from both rows are combined. (Right) Mask for the processed data.

the infrared standard star network consisting of $\mathrm{K}$ - and $\mathrm{M}$ giants (Cohen et al. 1999) and additional faint standard stars located around the north and south ecliptic poles (Reach et al. 2005; Ishihara et al. 2006b), which have a high visibility for the AKARI survey. The expected fluxes $\left(f_{\lambda}^{\text {quoted }}\left(\lambda_{i}\right)\right)$ of the standard stars at the effective wavelengths $(9 \mu \mathrm{m}$ and $18 \mu \mathrm{m})$ are calculated for the incident spectrum of $f_{\lambda} \propto \lambda^{-1}$ by convolving the model spectra of the standard stars $\left(f_{\lambda}(\lambda)\right)$ with the relative spectral response curves $\left(R_{i}\right)$ in electron units as

$f_{\lambda}^{\text {quoted }}\left(\lambda_{i}\right)=\frac{\int R_{i}(\lambda) \lambda f_{\lambda}(\lambda) \mathrm{d} \lambda}{\int\left(\frac{\lambda_{i}}{\lambda}\right) R_{i}(\lambda) \lambda \mathrm{d} \lambda}$,

where $i$ represents the band $i, \lambda_{i}$ is the effective wavelength of the band.

The fitting function to convert the measured signals $P$ into fluxes $F$ is given by

$\log (F)=\sum_{i=0}^{2} C_{i} \log (P)^{i}$,

where $C_{i}$ 's are fitting coefficients (their values are given in the release note). Finally, the derived conversion functions are applied to all the milli-seconds confirmed events. The zero magnitude flux is $56.26 \pm 0.8214 \mathrm{Jy}$ and $12.00 \pm 0.1751 \mathrm{Jy}$ for the $9 \mu \mathrm{m}$ and $18 \mu \mathrm{m}$ bands, respectively.

Figure 10 (top) shows the flux derived (by Eq. (10)) from the pipeline output as a function of the predicted in-band flux of the standard stars.

The accuracy of the calibrated flux is investigated by the ratio of the measured flux to the predicted flux of the standard stars. The results shown in the bottom panels of Fig. 10 indicate an accuracy for the absolute calibration of about $3 \%$ for the $9 \mu \mathrm{m}$ band and $4 \%$ for the $18 \mu \mathrm{m}$ band.

A systematic offset between A-type and K-M type giant standard stars is reported by Reach et al. (2005) for the calibration of the IRAC on Spitzer. We have only three A-type standard stars in our calibration for the $9 \mu \mathrm{m}$ band and no A-type stars are used for the calibration of the $18 \mu \mathrm{m}$ band due to the detection limit. No systematic offset is seen in the present calibration between A-type stars and K-M giants within the measurement uncertainties (Fig. 10). Note that the calibration of pointing observations of the IRC imaging mode does not show any appreciable offsets either (Tanabe et al. 2008).

To test the long-term stability of the photo response we investigated five standard stars that have been observed more than 30 times during the time of the survey. Figure 11 shows the ratio of the fluxes of individual measurements to the average fluxes of these stars as a function of time. From these data we deduce that the sensitivity is stable at the $\sim 2 \%$ level during the entire period of the observations.

Laboratory measurements of the filter transmission indicate possible blue leaks between the $3 \mu \mathrm{m}$ and $4 \mu \mathrm{m}$ for the $9 \mu \mathrm{m}$ band and between the $6 \mu \mathrm{m}$ and $7 \mu \mathrm{m}$ for the $18 \mu \mathrm{m}$ band. They are $0.01 \%$ at maximum and much smaller than the measurement errors. Thus the presence of the blue leaks is not confirmed. We calculated the predicted fluxes of the standard stars with and without the blue leak and confirmed that the difference is less than $0.1 \%$. We have verified that the blue leak of the $18 \mu \mathrm{m}$ band is negligible $(<1 \%)$ compared to the systematic errors by using asteroid calibrators whose flux can be well predicted (Müller \& Hasegawa, private communication).

\subsection{Catalog compilation}

The final list of the sources (hereafter point source catalog; PSC) has been prepared based on the following criteria. First, groups of multiple events located within a region of $5^{\prime \prime}$ radius are recognized as a same source. In this process, events in the 

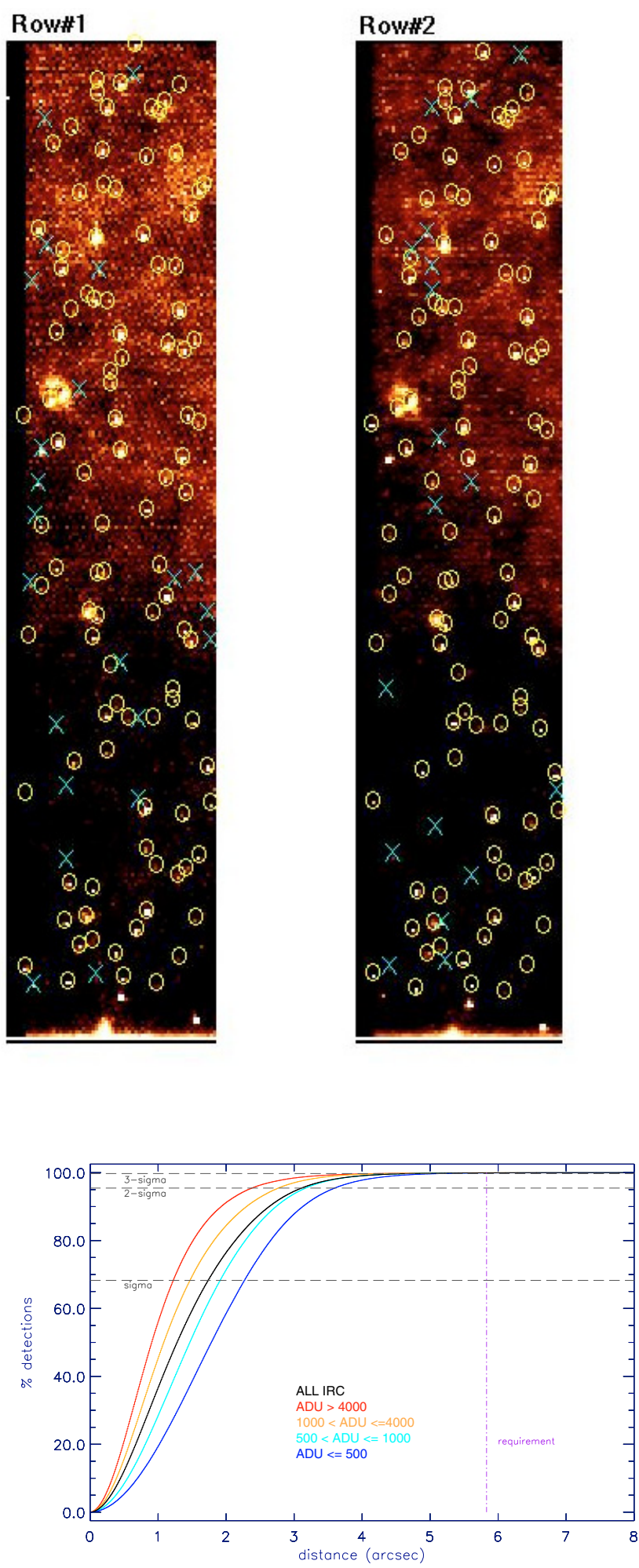

Fig. 9. Statistical error of the pointing reconstruction using $50 \%$ of the catalog data. The error is defined as the distance between the position determined by the pointing reconstruction and the position of the input reference catalog for events in the remaining 50\% data. The lines show the fraction of the IRC events with an error smaller than the given values. The color of the lines denotes the flux range of events.

south Atlantic anomaly (SAA) are excluded. Only groups containing at least two events are recognized as an actual celestial source. After the first grouping process, the distance from a source candidate to the nearest one is investigated, and if there

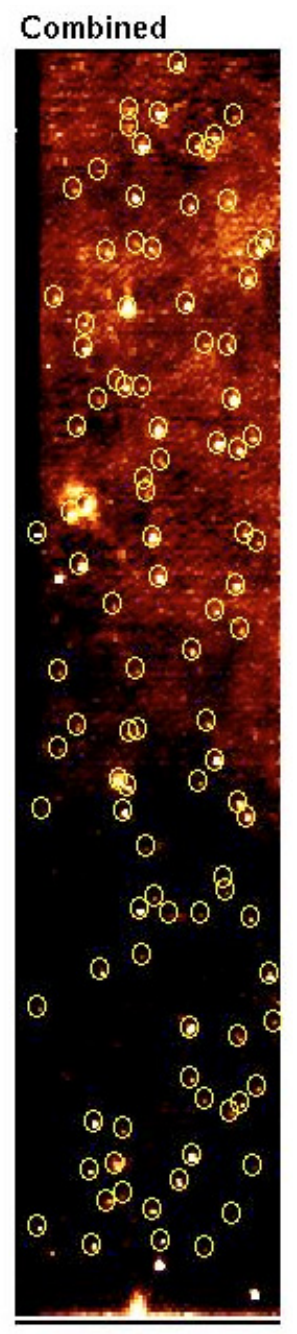

Fig. 8. Example of the event detection and milli-seconds confirmation. The left image is obtained by row\#1 and the center image is obtained by row\#2. The right image is constructed by combining data from two rows. The crosses mark events extracted on a single row image, but rejected in milli-seconds confirmation. The circles show events extracted on both rows with similar fluxes, which are thus confirmed.

are two or more groups within $7^{\prime \prime}$, we consider them as a single source associated with outskirts events. In this case we take the group with the maximum number of events as a source candidate and discard the other groups. Then the source lists in the $9 \mu \mathrm{m}$ and $18 \mu \mathrm{m}$ band are merged into a single list. Sources within $7^{\prime \prime}$ are regarded as the same source in both bands. The position (RA, Dec) and associated position error (the major and minor axes and the position angle) are calculated from the events in the $9 \mu \mathrm{m}$ band only, if the number of available events is larger than or equal to 2 . Otherwise, these data are calculated from the events in the $18 \mu \mathrm{m}$ band only. The flux and associated error of the source are estimated from the mean and the mean error of multiple measurements of the events, respectively. Event data near the edge of the image strips are excluded from the flux calculation unless the exclusion leaves only zero or one event.

The numbers of the events obtained in each step of the process are summarized in Table 2. The spatial distribution of the rejected events (those without hours and months confirmation) is shown in Fig. 12. Most of the rejected events are ascribed to asteroids, geostationary satellites, and the high-energy particle hits in the SAA. These objects are recognized as such because of their spatial distribution and characteristic features. It should be noted that the actual sky coverage for this catalog is smaller than the value quoted in Sect. 2.3; this is due to the severe condition of $N_{\text {events }} \geq 2$ as well as to the exclusion of all data affected by the SAA. 

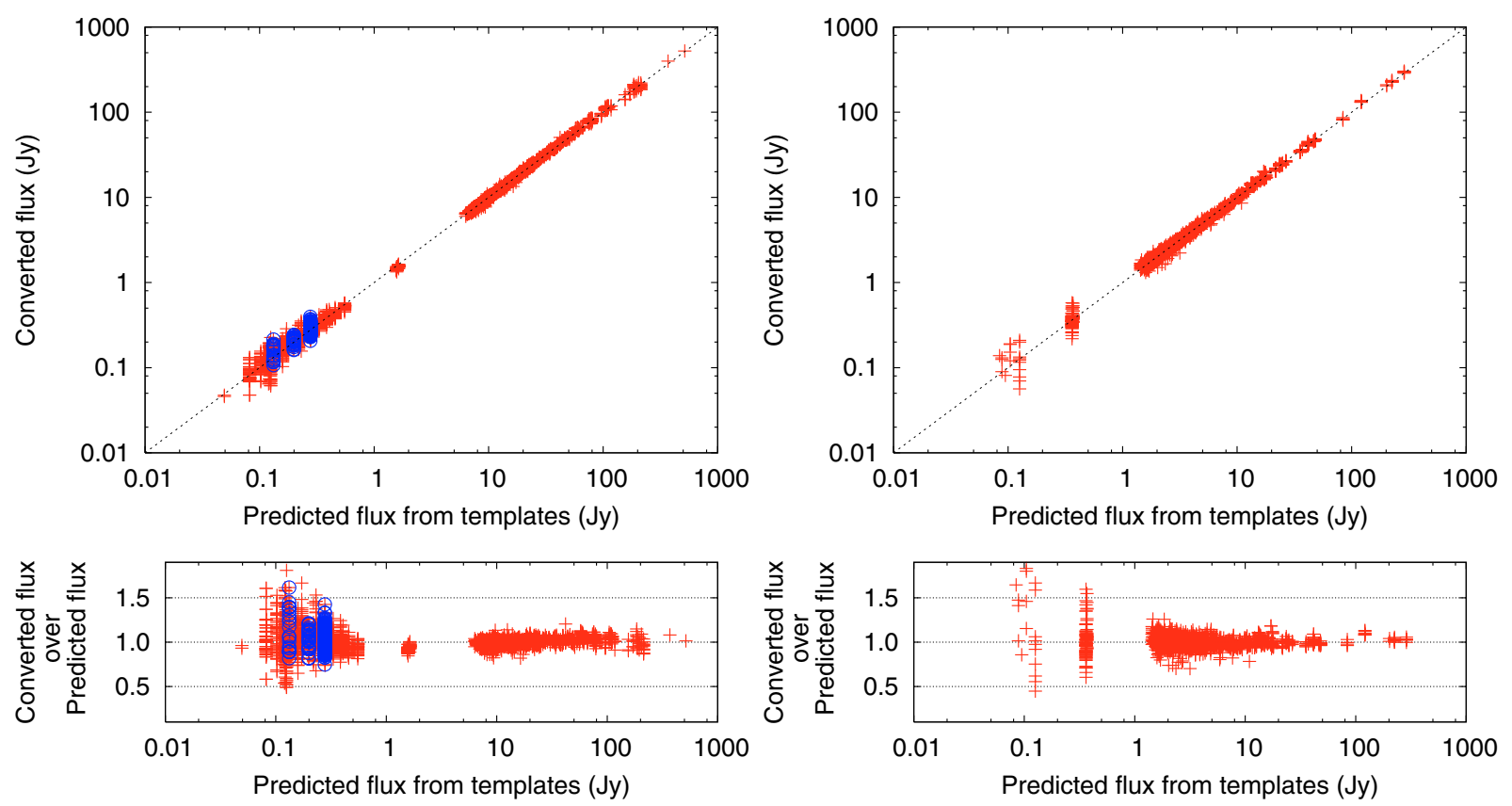

Fig. 10. (Top left) Calibrated fluxes obtained from the pipeline output through Eq. (10) are plotted as a function of the predicted in-band fluxes of the standard stars in the $9 \mu \mathrm{m}$ band. The slid line indicates $y=x$ for reference. (Bottom left) Ratio of the measured to predicted fluxes for the standard stars. The right panels show the same plots for the $18 \mu \mathrm{m}$ band. The symbols and lines are the same as in the left panels. The blue points show the three A-type stars used for the $9 \mu \mathrm{m}$ band calibration. All standard stars used for the $18 \mu \mathrm{m}$ band calibration are K-M giants, and no A-type stars were used.

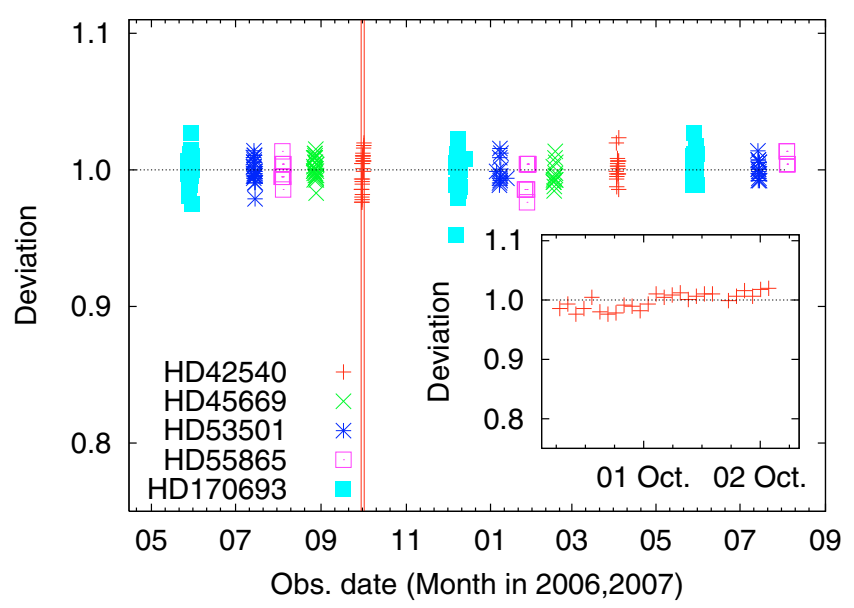

Fig. 11. Ratio of the measured fluxes to the average fluxes as a function of observing time for five bright $(>1 \mathrm{Jy})$ standard stars observed at $9 \mu \mathrm{m}$ more than 30 times during the survey. The stars used and the corresponding $9 \mu \mathrm{m}$ fluxes and number of detections are: HD 42540 (plus, $8.33 \mathrm{Jy}, 44$ times), HD 45669 (cross, 9.79 Jy, 43 times), HD 53501 (star, $9.30 \mathrm{Jy}, 45$ times), HD 55865 (open box, $16.7 \mathrm{Jy}, 33$ times) and HD 170693 (filled box, $9.60 \mathrm{Jy}, 76$ times), respectively. The closeup shown in the right bottom panel is to check for the temporal variation on a short timescale.

\section{Evaluation of the catalog}

\subsection{Spatial distribution}

Figure 13 shows the spatial distribution of the number density of the cataloged sources in the $9 \mu \mathrm{m}$ and $18 \mu \mathrm{m}$ bands after removal of the rejected events. One can easily recognize the highly populated regions, which correspond to the Galactic plane, the Large Magellanic Cloud (LMC), the Small Magellanic Cloud (SMC), and the nearby star forming regions like $\rho$ Oph, Orion

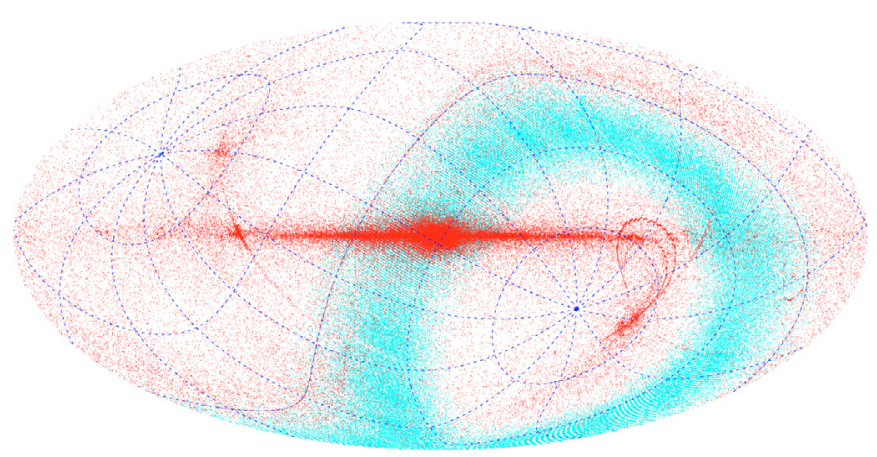

Fig. 12. Spatial distribution in an Aitoff Galactic coordinates projection of the $18 \mu \mathrm{m}$ events rejected in the catalog compilation process. The grids indicate the equatorial coordinates. Most of the events correspond to high energy particles due to the south Atlantic anomaly (SAA), or to moving objects like asteroids and comets (aligned around $|\beta|<10^{\circ}$ ), to geostationary satellites $\left(|\mathrm{Dec}| \sim 0^{\circ}\right)$, and to retired geostationary satellites $\left(|\mathrm{Dec}|<10^{\circ}\right)$. The color version will be available in the online version, in which the SAA events are plotted in cyan, and the remaining objects in red.

and Taurus. Note that the $18 \mu \mathrm{m}$ sources are strongly concentrated near the thick Galactic plane, whereas the $9 \mu \mathrm{m}$ source counts fall off smoothly with the galactic latitude.

\subsection{Flux accuracy}

The histogram in Fig. 14 shows the relative flux errors in the $9 \mu \mathrm{m}$ and $18 \mu \mathrm{m}$ bands as a function of flux. As expected, the largest errors are associated with sources with the lowest fluxes. We find that the most probable error is $2-3 \%$ and the probability of larger errors is small. The relative errors are smaller than $15 \%$ for $80 \%$ of the sources and smaller than $30 \%$ for $96 \%$ of the sources. It should be stressed that at this stage we cannot 

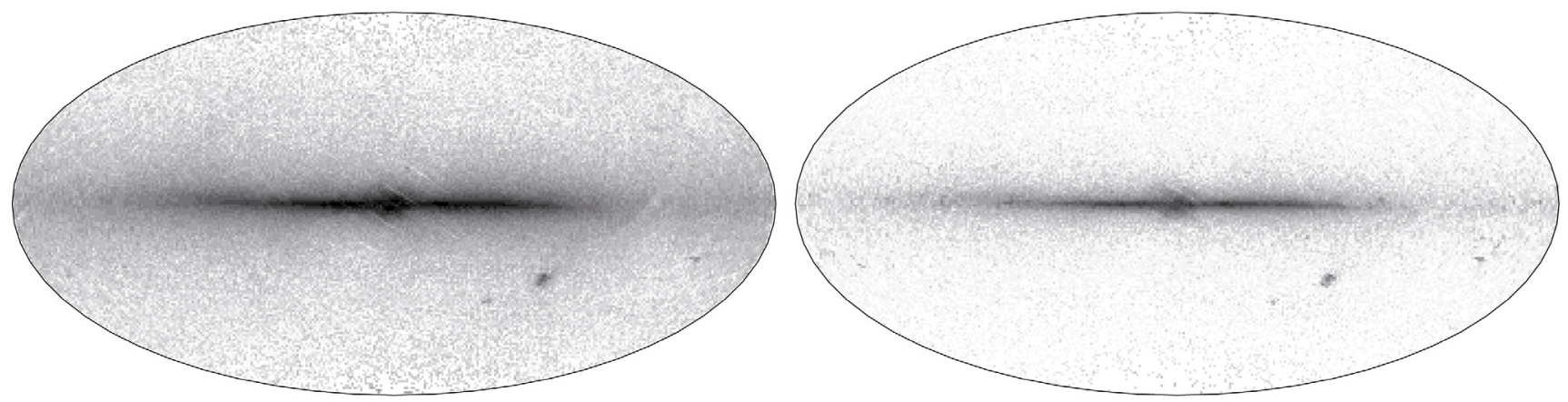

Fig. 13. Spatial number density distribution of the detected sources in the Galactic coordinates of the Aitoff projection. (Left) $9 \mu \mathrm{m}$ sources of 851189 and (right) $18 \mu \mathrm{m}$ sources of 195893 .
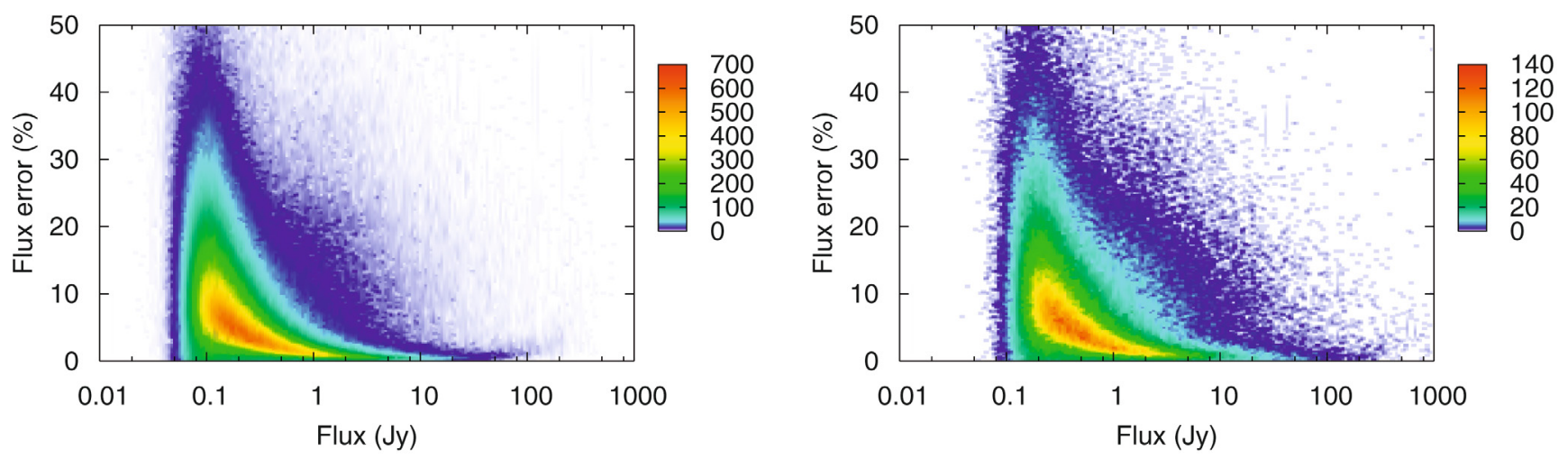

Fig. 14. Relative flux errors (\%) for $9 \mu \mathrm{m}$ (left) and $18 \mu \mathrm{m}$ sources (right) as a function of flux plotted in the density maps.

Table 2. Number of events at each step of the process for the preparation of the PSC.

\begin{tabular}{lrr}
\hline \hline & $9 \mu \mathrm{m}$ & $18 \mu \mathrm{m}$ \\
\hline Detected & 16752471 & 9655059 \\
Milli-seconds confirmed & 4929586 & 1300945 \\
Hours or months confirmed & $851189^{*}$ & $195893^{*}$ \\
Band merged & \multicolumn{2}{c}{$877091^{*}$} \\
\hline
\end{tabular}

Notes. ${ }^{(*)}$ - Current values. The number of sources may be updated in further catalog releases.

quantify how much variable objects "artificially" contribute to an increase of the scatter in the observed fluxes. The inclusion of mid-IR variability of the objects will be considered in future catalog releases.

Figure 15 shows a typical signal-to-noise ratio $(S / N)$ associated with the different flux levels. The figure indicates that the expected $S / N$ is $\sim 6$ for the faintest sources of $\sim 0.045 \mathrm{Jy}$ in the $9 \mu \mathrm{m}$ band, while it is $\sim 3$ for $\sim 0.06$ Jy sources in the $18 \mu \mathrm{m}$ band. The figure also shows that the $S / N$ increases with increasing flux, but above $0.6 \mathrm{Jy}$ and $0.9 \mathrm{Jy}$ it becomes constant or slightly decreases to $\sim 20$ and $\sim 15$ for $9 \mu \mathrm{m}$ band and $18 \mu \mathrm{m}$ band, respectively. This leveling-off of the $S / N$ is due to uncertainties in the data reduction process. Errors in the correction for the detector reset anomaly, linearity, and flat-fielding limit accuracy of the flux measurement.

\subsection{Position accuracy}

A test of the AKARI position accuracy is indicated in Fig. 16, which shows the angular separation between AKARI sources and the nearest 2MASS source (Cutri et al. 2003). The figure indicates that about $73 \%$ of the sources have an angular separation $<1^{\prime \prime}$, nearly $95 \%$ of the sources have a separation $<2 "$. On average, the mean separation between AKARI and 2MASS coordinates of matching sources is $0.8 \pm 0.6^{\prime \prime}$.

\subsection{Number of detections per source}

The histogram in Fig. 17 shows the number of detections per source, both in differential and integrated counts. As described in Sect. 3.5, all the cataloged sources are detected at least twice. Over $80 \%$ of the sources have more than three detections and about half of the sources have more than five detections (right side scale of Fig. 17). The average number of detections of the cataloged sources is six.

\subsection{False detections and missing sources}

False detections. False detections are in principle rejected in the two-step source confirmation process. However, there is an off-chance of including (1) high energy particle hit events; (2) slow moving objects; (3) ghosts produced in the camera optics; and (4) those triggered by the signal saturation.

Missing bright sources. Some of the bright sources which have IRAS measurements are not included in the catalog because they are (1) not recognized as a point source with the beam size of AKARI because of the spatial extension (Fig. 18 shows histograms of the spatial size of the AKARI/MIR sources); (2) located in the area not covered by this survey; or (3) observed only once. A total 1722 IRAS sources with the good quality flag 

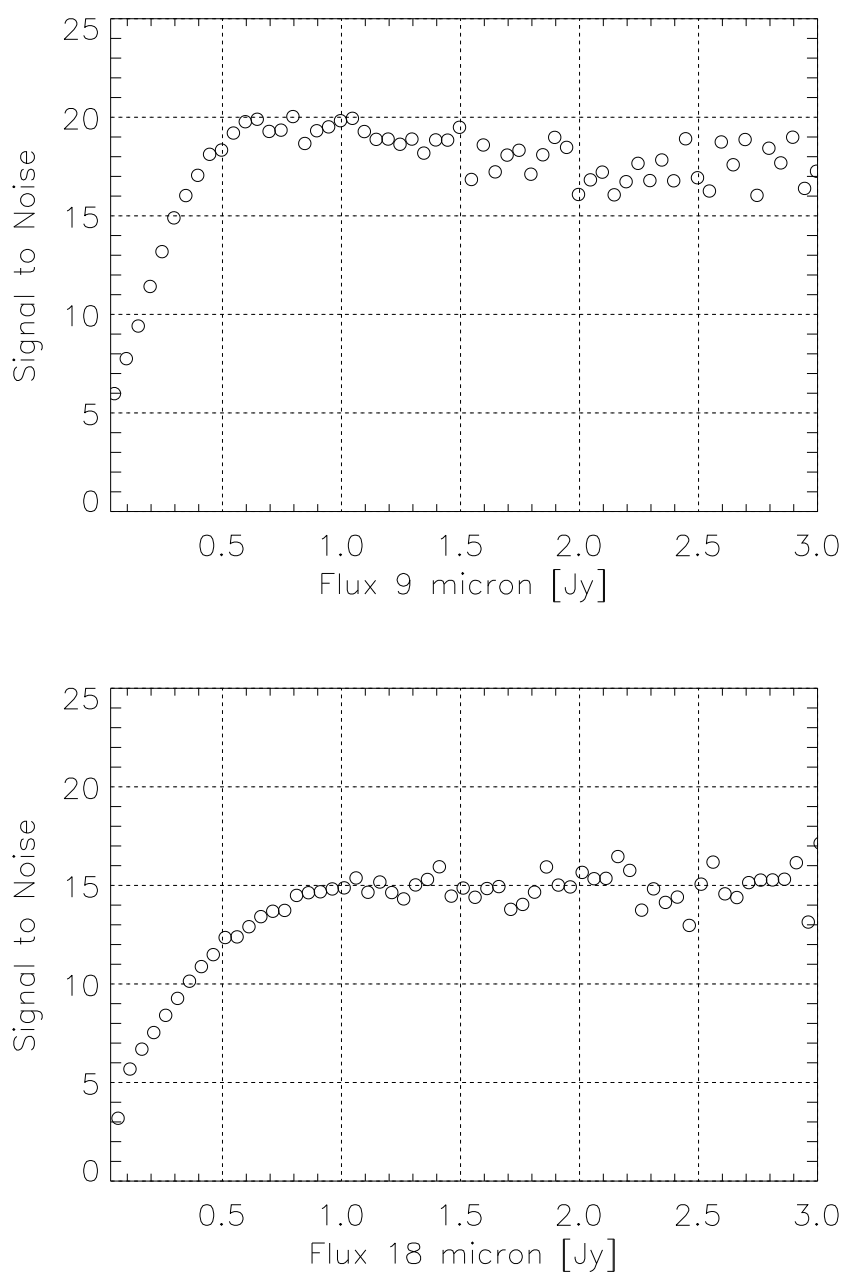

Fig. 15. Signal-to-noise ratio as a function of the $9 \mu \mathrm{m}$ (top) and $18 \mu \mathrm{m}$ band flux (bottom).

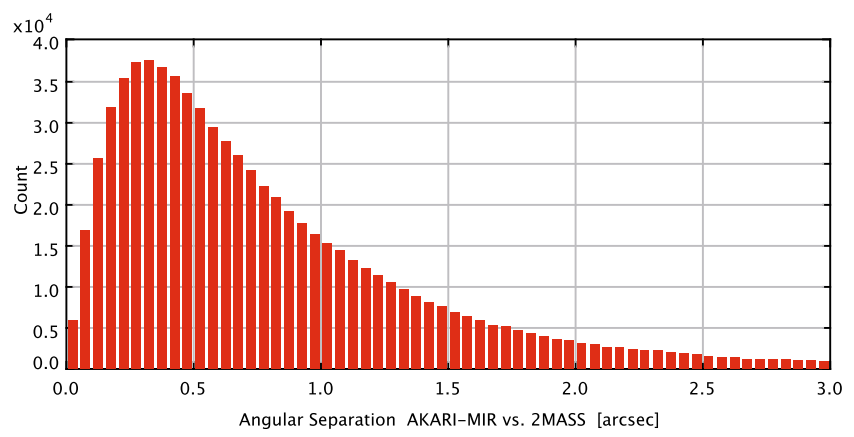

Fig. 16. Histogram of the angular separation between the AKARI coordinates and the 2MASS coordinates for the common sources.

$\left(f_{\text {qual12 }}=2\right.$ and $f_{\text {qual25 }}=3$ ) are not included in both the $9 \mu \mathrm{m}$ and $18 \mu \mathrm{m}$ sources.

\subsection{Source counts versus flux}

It is instructive to compare the overall distribution of the source counts between the AKARI and IRAS PSCs. The histograms of the source counts in the two surveys are compared in Fig. 19. The large difference in terms of the lower cutoff flux in the source counts between the two surveys is a clear signature of the

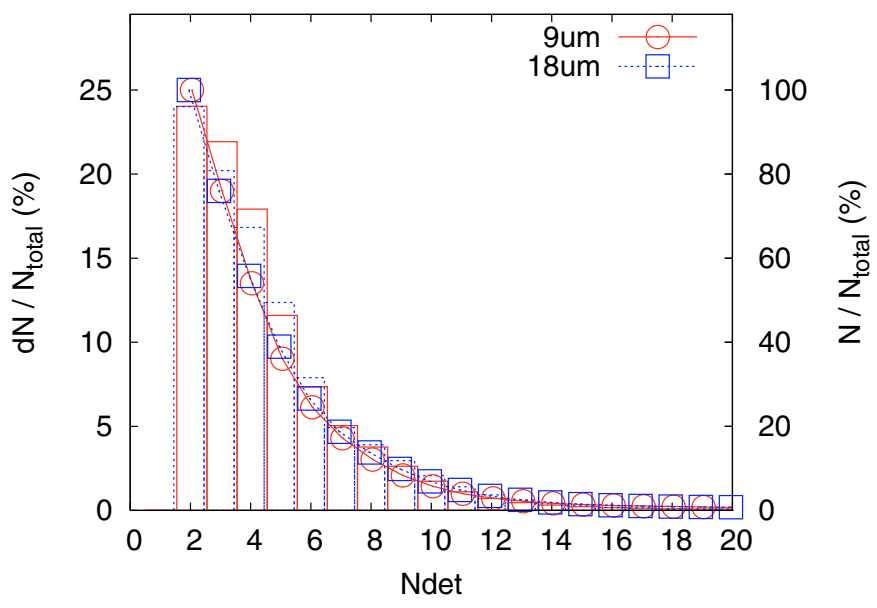

Fig. 17. Number of detections per source at $9 \mu \mathrm{m}$ (solid lines) and $18 \mu \mathrm{m}$ source (dotted lines). The differential counts are shown as histograms in the scale on the left vertical axis and the integrated counts are shown by the lines with symbols in the scale on the right vertical axis.

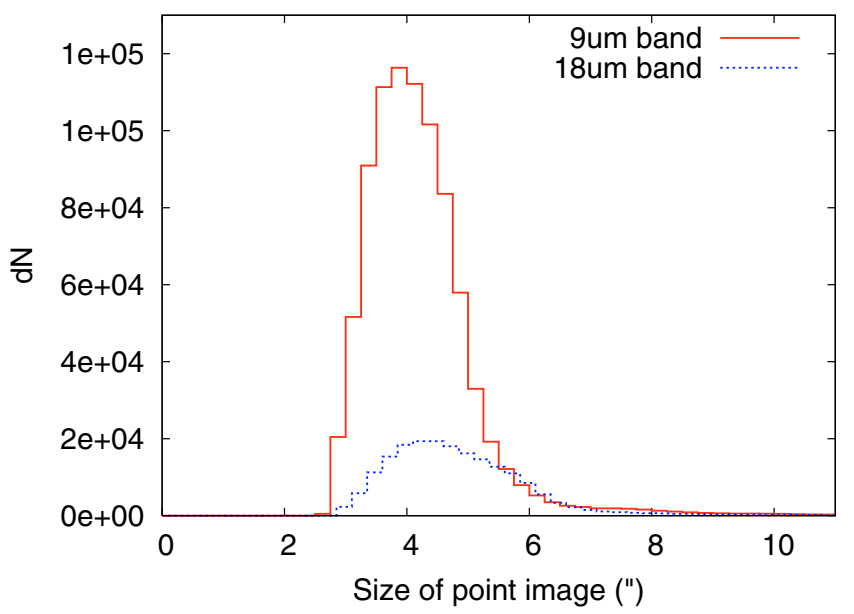

Fig. 18. Histograms of the spatial extension of AKARI sources at $9 \mu \mathrm{m}$ and $18 \mu \mathrm{m}$, (solid and dashed lines, respectively). The spatial extension is calculated as $(a+b) / 2$, where $a$ and $b$ are the length in arcsecond for the major axis and the minor axes of the source image, respectively.

considerably higher sensitivity, up to a factor of nearly 10 , of the AKARI survey compared to IRAS.

The distribution of source counts of the subsets of the $9 \mu \mathrm{m}$ sources with and without a $18 \mu \mathrm{m}$ counterpart are shown in the left panel of Fig. 20. Similarly that for the $18 \mu \mathrm{m}$ sources with and without a $9 \mu \mathrm{m}$ counterpart is shown in the right panel of Fig. 20. Most of the $18 \mu \mathrm{m}$ sources have $9 \mu \mathrm{m}$ detections, whereas fainter $9 \mu \mathrm{m}$ sources do not have their $18 \mu \mathrm{m}$ counter parts.

\subsection{Completeness}

The completeness of a survey above a given flux level is usually defined as the fraction of true sources that can be detected above that level. It is difficult to apply this concept to the AKARI survey because one should dispose of a statistically significant sample of true sources with known IRC fluxes. The standard stars used for the AKARI/IRC PSC calibration might not represent a statistically significant sample in view of the rather poor coverage at low flux levels. 
D. Ishihara et al.: AKARI MIR all-sky survey
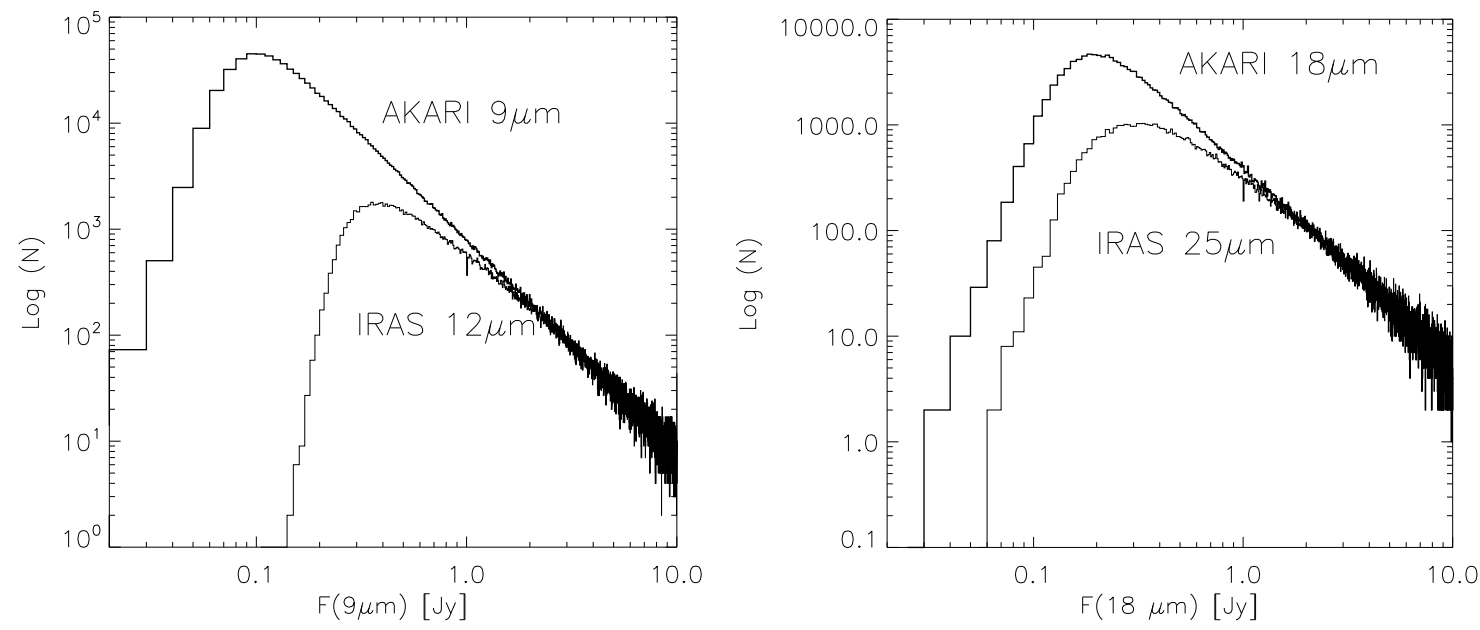

Fig. 19. The distribution of source counts as a function of the $9 \mu \mathrm{m}$ and $18 \mu \mathrm{m}$ flux for AKARI is compared with that from the IRAS survey at $12 \mu \mathrm{m}$ and $25 \mu \mathrm{m}$.
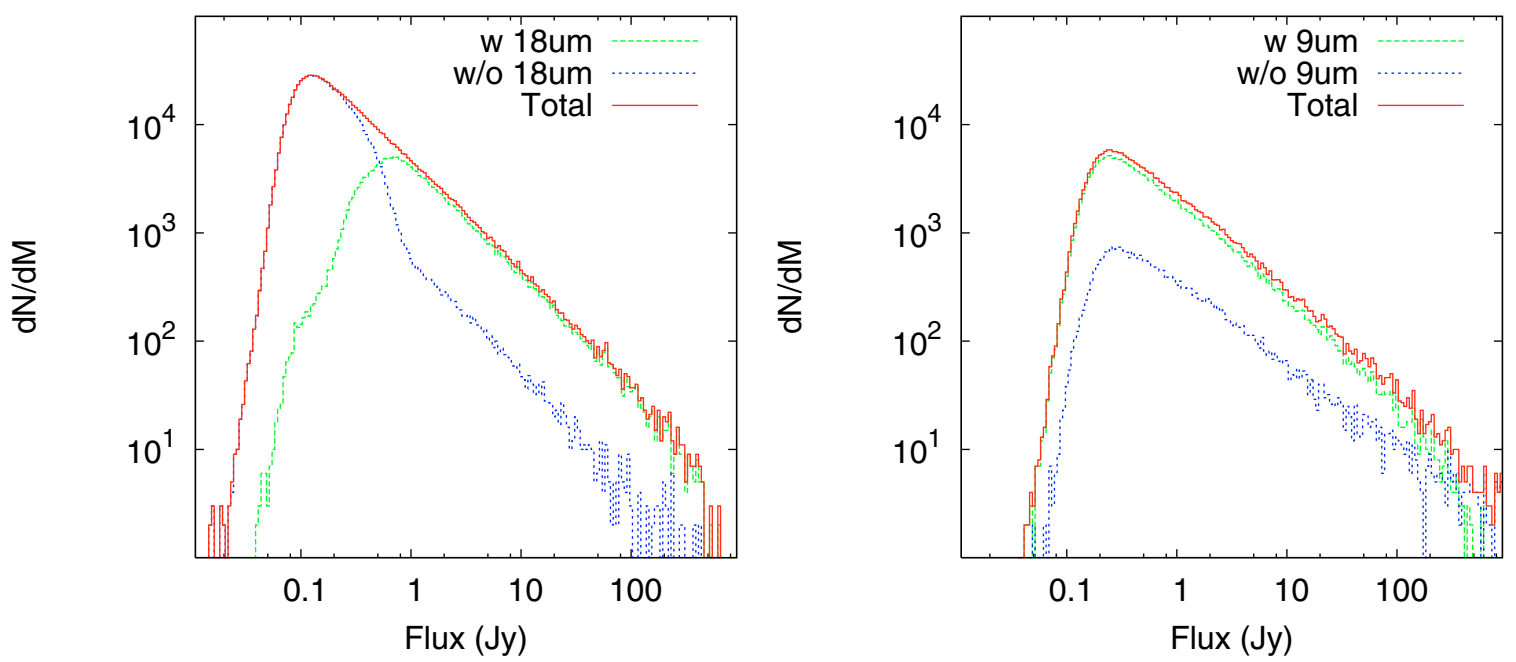

Fig. 20. Flux distributions of the detected sources. (Left) Flux distribution for the $9 \mu \mathrm{m}$ sources with $18 \mu \mathrm{m}$ detections (dashed), without $18 \mu \mathrm{m}$ detections (dotted) and total (solid). (Right) Flux distribution for the $18 \mu \mathrm{m}$ sources with $9 \mu \mathrm{m}$ detections (dashed), without $9 \mu \mathrm{m}$ detections (dotted) and total (solid).

To assess the completeness of the AKARI-MIR survey we have thus taken a different approach based on the distribution of sources according to their flux. Figure 19 shows an interesting feature: source counts decline exponentially with increasing flux after a peak value is reached around $0.1 \mathrm{Jy}(9 \mu \mathrm{m}$ band) and $0.2 \mathrm{Jy}$ (18 $\mu \mathrm{m}$ band). One can thus make a reasonable assumption that the exponential decay is an intrinsic property of source counts at the relevant wavelengths and, on this basis, one can define completeness as the ratio of the number of sources actually observed by the number of sources predicted by the above equations. The results of completeness are shown in Fig. 21. From this figure we deduce the completeness ratios reported in Table 3.

To evaluate the detection limit of the survey, we can make use of the $S / N$ characteristics shown in Fig. 15. From this figure one can deduce that for $S / N \sim 5$ the detection limit is about 0.05 and $0.09 \mathrm{Jy}$ in the $9 \mu \mathrm{m}$ and $18 \mu \mathrm{m}$ bands, respectively.

A summary of the completeness of the survey at various flux levels together with the corresponding values of the $S / N$ ratio are given in Table 3 . These results agree fairly well with a prelaunch prediction in Ishihara et al. (2006a).
Table 3. Completeness and signal-to-noise ratio.

\begin{tabular}{lrrrr}
\hline \hline \multicolumn{5}{c}{$9 \mu \mathrm{m}$ band } \\
\hline Completeness & $5 \%$ & $50 \%$ & $80 \%$ & $100 \%$ \\
Flux[Jy] & 0.07 & 0.10 & 0.11 & 0.15 \\
$S / N$ & 6.7 & 7.6 & 8.5 & 9.8 \\
\hline & $18 \mu \mathrm{m}$ band & & \\
Completeness & $5 \%$ & $50 \%$ & $80 \%$ & $100 \%$ \\
Flux[Jy] & 0.10 & 0.16 & 0.21 & 0.28 \\
$S / N$ & 5.0 & 6.5 & 7.6 & 8.7 \\
\hline
\end{tabular}

The completeness is also tested separately for different Galactic latitude ranges, $|b|<2.2^{\circ}, 2.2^{\circ}<|b|<8.3^{\circ}$, and $|b|>8.3^{\circ}$, where the division is made to have roughly similar numbers of the objects. Figure 21 indicates that the completeness becomes worse in the Galactic plane $(|b|<2.2$ degree), which can be attributed to either the source confusion or the spatially variable background. 

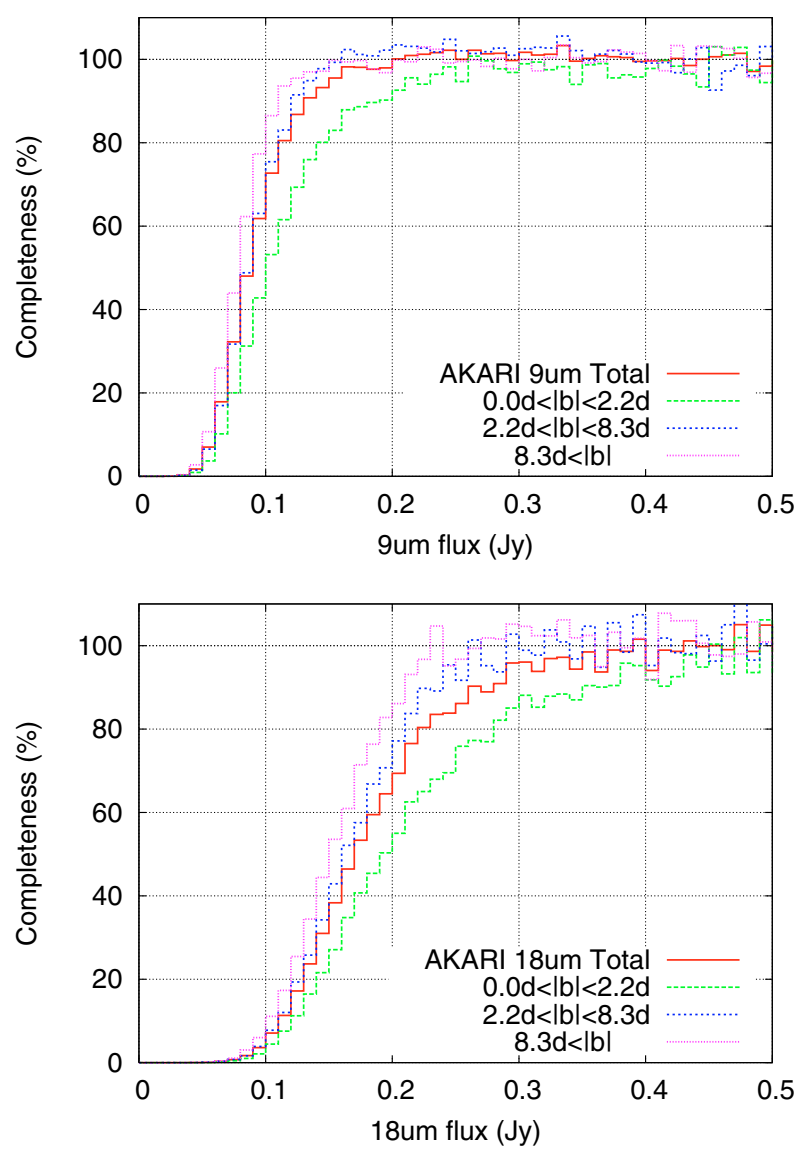

Fig. 21. Completeness ratio of the AKARI/IRC survey in the $9 \mu \mathrm{m}$ and $18 \mu \mathrm{m}$ bands. Plots are made also for each galactic regions, $|b|<2.2^{\circ}$, $2.2^{\circ}<|b|<8.3^{\circ}$, and $|b|<8.3^{\circ}$, separately.

\subsection{Comparison with the IRAS catalog}

To further evaluate the reliability of the AKARI flux measurements at $9 \mu \mathrm{m}$ and $18 \mu \mathrm{m}$, we have carried out a comparison of these with the IRAS $12 \mu \mathrm{m}$ and $25 \mu \mathrm{m}$ fluxes for common sources.

In Fig. 22 a comparison is given between AKARI and IRAS fluxes observations for the cross-identified objects. It is evident that there is a close correlation between the two sets of data. This is especially true for stellar sources; indeed, many MIR sources like compact $\mathrm{H}$ II regions, reflection nebulae and planetary nebulae, have extended emission or are located within extended objects. In such cases, IRAS measures the total flux of the extended emission because of the larger apertures $(0.75 \times 4.5-4.6$ pixel size), whereas AKARI measures the flux of the peak emission on the extended objects with a smaller aperture $\left(\sim 9^{\prime \prime}\right.$ beam size $)$ as demonstrated in the case of a reflection nebula (Ishihara et al. 2007). Some sources are brighter in the IRAS PSC than in the AKARI PSC. The difference could be attributed to the effect of the difference in the spatial resolution.

\section{Summary}

The AKARI mid-infrared all-sky survey was performed with two mid-infrared broad bands centered at $9 \mu \mathrm{m}$ and $18 \mu \mathrm{m}$. More than $90 \%$ of the entire sky was observed in both bands. A total of 877091 sources (851 189 for $9 \mu \mathrm{m}, 195893$ for $18 \mu \mathrm{m}$ ) are detected and included in the present release of the point source catalog. This AKARI mid-infrared point source catalog is scheduled for public release in 2010 after the prioritized period for the team members (Release Note 2009). We present the spatial distribution, flux distribution, flux accuracy, position accuracy, and completeness of the sources in the AKARI MIR all-sky survey catalog version $\beta-1$.

The AKARI mid-infrared survey provides a unique data-set relevant to interstellar, circumstellar-, and extra galactic astronomy. This new deep, large survey is well suited to research in the various fields of astronomy, like the search for warm debris disks, of asteroid analog (Fujiwara et al. 2009a,b), and provides valuable information for the study of planet formation and other fields. The chemical compositions of an unprecedented number of dust-forming asymptotic giant branch (AGB) stars can also be investigated (Ita et al. 2009), making use of the characteristics of the filter bands of this survey. These studies enable new discussions on the structure, the star formation history and the cycle of matter in our Galaxy. The mid-infrared all-sky survey also provides a chance to detect highly obscured active galactic nuclei (AGN), which are difficult to observe in previous optical or X-ray surveys. The population of obscured AGN is expected to contribute to the hard X-ray background, which is not completely resolved into individual source yet (Ueda et al. 2003).

Acknowledgements. This research is based on observations with AKARI, a JAXA project with the participation of ESA. This research has made use of the SIMBAD database, operated at CDS, Strasbourg, France. This work was supported by the the Nagoya University Global COE Program, "Quest for Fundamental Principles in the Universe (QFPU)" from JSPS and MEXT of Japan. This work was supported by a Grant-in-Aid for Scientific Research on Priority Areas from the Ministry of Education, Culture, Sports, Science and Technology, Japan (No. 16077201). H.F. is financially supported by the Japan Society for the Promotion of Science. S.H. was supported by Space Plasma Laboratory, ISAS, JAXA.

\section{References}

Aumann, H. H., Beichman, C. A., Gillett, F. C., et al. 1984, ApJ, 278, 23 Bertin, E., \& Arnouts, S. 2000, A\&A, 117, 393

Beichman, C. A., Neugebauer, G., Habing, H. J., Clegg, P. E., \& Chester,

T. J. 1988, Infrared astronomical satellite (IRAS) catalogs and atlases, Explanatory supplement, 1

Cohen, M., Walker, R. G., Carter, B., et al. 1999, AJ, 117, 1864

Cutri, R. M., Skrutskie, M. F., van Dyk, S., et al. 2003, The IRSA 2MASS AllSky Point Source Catalog, NASA/IPAC Infrared Science Archive, http: // irsa.ipac.caltech. edu/applications/Gator/

Fujiwara, H., Yamashita, T., Ishihara, D., et al. 2009a, ApJ, 695, L88 Fujiwara, H., Ishihara, D., Kataza, H., et al. 2009b, A\&A, submitted Ishihara, D., Wada, T., Watarai, H., et al. 2003, Proc. SPIE, 4850, 1008 Ishihara, D., Wada, T., Onaka, T., et al. 2006a, PASP, 118, 324 Ishihara, D., Onaka, T., Kataza, H., et al. 2006b, AJ, 131, 1074 Ishihara, D., Onaka, T., Kaneda, H., et al. 2007, PASJ, 59, S443 Ita, Y., Matsuura, M., Ishihara, D., et al., 2010, A\&A, 514, A2 Kaneda, H., Kim, W., Onaka, T., et al. 2007, PASJ, 59, S423

AKARI/IRC team, Kataza, H., Alfageme, C., Cassatella, A., et al. 2009, AKARI/IRC All-Sky Survey Bright Source Catalog Version $\beta-1$ Release Note Rev.1

Kawada, M., Baba, H., Barthel, P. D., et al. 2007, PASJ, 59, S389 Murakami, H., Baba, H., Barthel, P., et al. 2007, PASJ, 59, S369

Neugebauer, G., Habing, H. J., van Duinen, R., et al. 1984, ApJ, 278, 1 Onaka, T., Matsuhara, H., Wada, T., et al. 2007, PASJ, 59, S401

Price, S. D., Egan, M. P., Carey, S. J., Mizuno, D. R., \& Kuchar, T. A. 2001, AJ, 121,2819

Reach, W. T., Megeath, S. T., Cohen, M., et al. 2005, PASP, 117, 978

Soifer, B. T., Sanders, D. B., Madore, B. F., et al. 1987, ApJ, 320, 238

Takita, S., Kitamura, Y., Kataza, H., et al. 2009, PASJ, 61, 291

Tanabé, T., Sakon, I., Cohen, M., et al. 2008, PASJ, 60, 375

Wada, T., Fujishiro, N., Ishihara, D., et al. 2003, Proc. SPIE, 4850, 179

Ueda, Y., Akiyama, M., Ohta, K., \& Miyaji, T. 2003, ApJ, 598, 886 

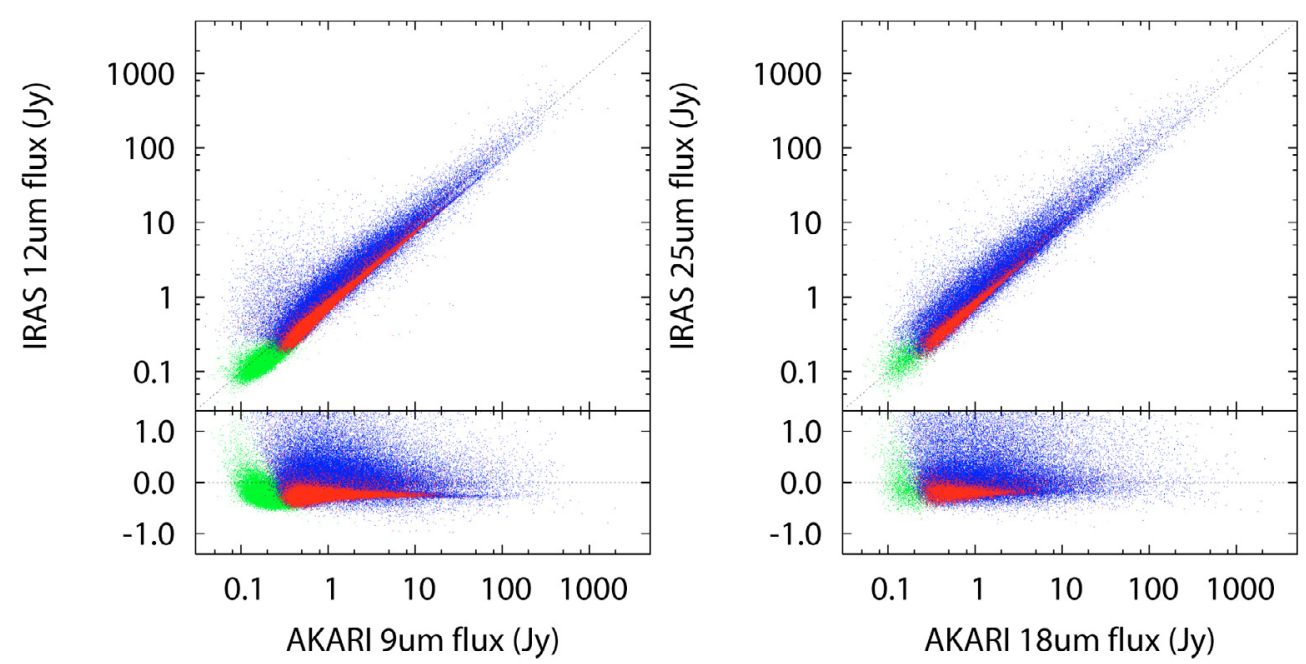

Fig. 22. (Left) Comparison of the AKARI $9 \mu \mathrm{m}$ versus IRAS $12 \mu \mathrm{m}$ fluxes for the cross-identified sources. The red dots indicate sources with $f_{\text {qual } 12}=3$ in the IRAS PSC labeled as a star in the SIMBAD database. The blue dots show the other sources with $f_{\text {qual } 12}=3$ in the IRAS PSC. The green dots indicate IRAS FSC sources. (Right) Comparison of the AKARI $18 \mu \mathrm{m}$ versus IRAS $25 \mu \mathrm{m}$ fluxes for the cross-identified sources. The symbols are the same as in the left panel.
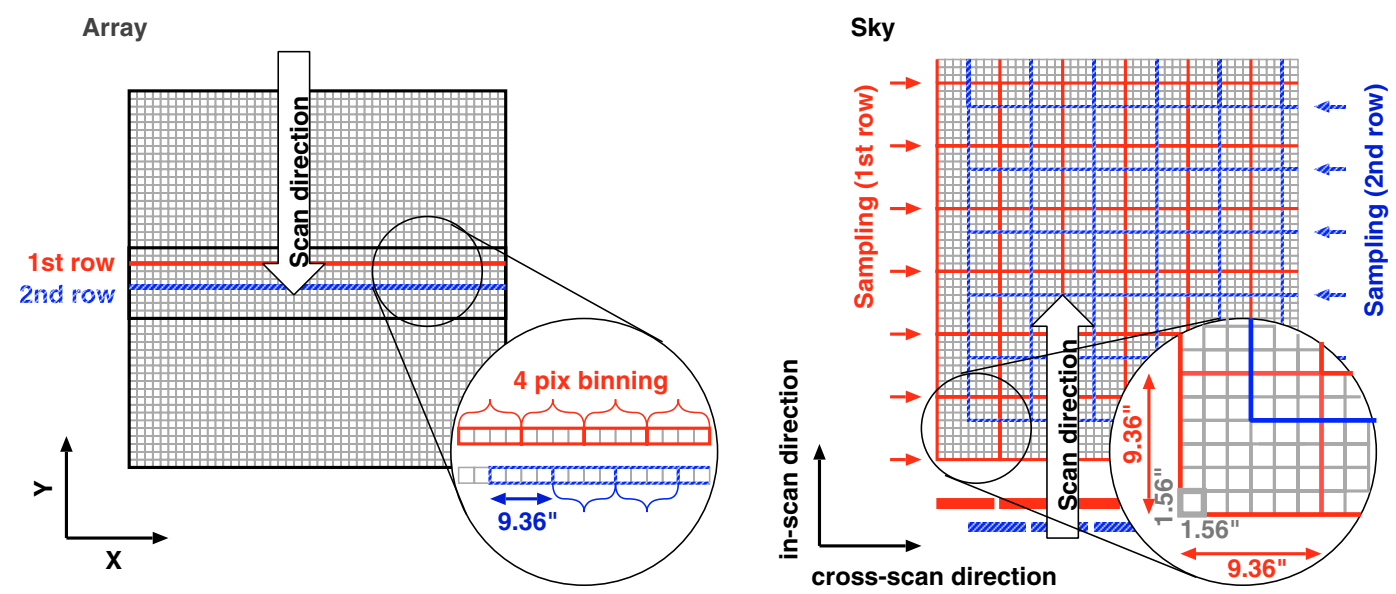

Fig. A.1. (Left) Illustration of the nest $4 \times 4$ mode array operation in the survey mode. (Right) Image reconstruction of the data taken in the nest $4 \times 4$ mode operation.
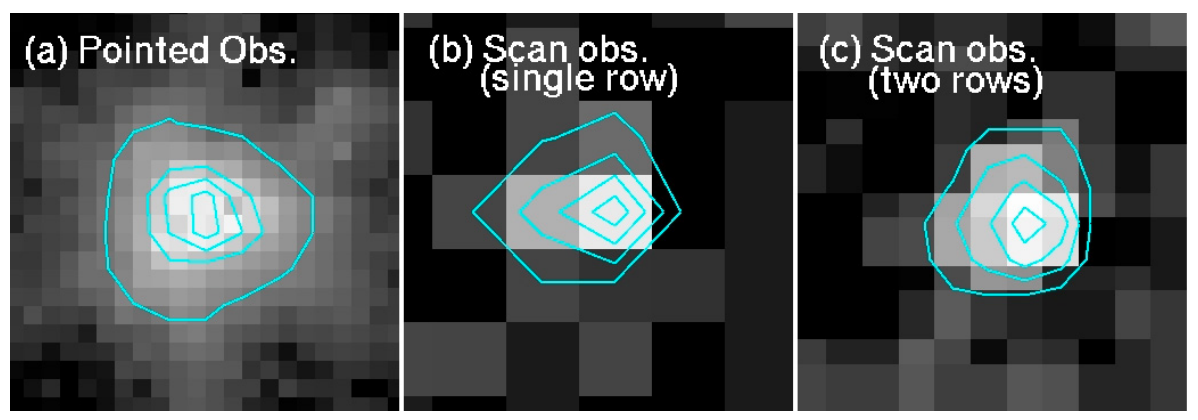

Fig. A.2. $9 \mu \mathrm{m}$ image of the standard star (HD 42525): a) the full-resolution image obtained in the pointed observation, b) the images processed from the single-row data in the survey observation, and c) the images reconstructed from the two-row data in the survey observation.

\section{Appendix A: Nest $4 \times 4$ mode operation and unit image construction}

Here we explain in more detail of the data acquisition in the allsky survey mode and subsequent image reconstruction method to describe the pixel scale (Fig. A.1). Though the original pixel scale of the MIR-S camera is 2 '. $34 \times 2$ '. $34(2$ '. $51 \times 2$ '. 39 for MIR-L), we degrade the spatial resolution in the survey observation to reduce the amount of output data to meet the downlink capacity. The pixel scale of the data taken by a single row in the in-scan direction is adjusted to 9.36 by setting the sampling rate as $22.7 \mathrm{~Hz}$ under the scan rate of the satellite of $216^{\prime \prime} \mathrm{s}^{-1}$. The pixel scale in the cross-scan direction is adjusted as 9.'36 by binning of the output of four adjacent pixels. The resulting effective pixel scale obtained in the scan by a single row (hereafter virtual pixel scale) is four times larger than the original pixel scale. The scan observation was made by two rows to enable two independent observations in the milli-seconds interval. The sampling timing and the combination of the binning pixels are adjusted to construct two independent grids on the sky. Because the scan 
rate was changed from the value expected before launch due to the change in the altitude from the planned $750 \mathrm{~km}$ to $700 \mathrm{~km}$, the shift of the grids in the in-scan direction is not exactly a half of the virtual pixel size.

Figure A.2 compares an image of the same star constructed from a single-row observation in the survey mode to one processed from a two-row observation in the survey mode, and one obtained in the imaging mode in the pointed observation with the original pixel scale. The FWHM of the PSF for the $9 \mu \mathrm{m}$ and $18 \mu \mathrm{m}$ bands is $5^{\prime \prime} .5$ and $5^{\prime \prime} .7$, respectively. The virtual pixel size is larger than the PSF size. However, the process of the two-row observations in the survey mode reconstructs the image with the spatial resolution compatible with that obtained in the pointed observations with the original pixel size (center of Fig. A.2).

Thus the process of the two-row observation allows us to make (1) a confirmation of the source detection in milli-seconds after (2) reduction in the output data rate and (3) higher spatial resolution than that in the single-row operation. The apparent asymmetry seen in the pointed data (Fig. A.2a) can be attributed to the effect of the telescope truss. Part of the asymmetry seen in the survey data may also be attributed to the under-sampling operation. No variation of the PSFs is recognized in the $9 \mu \mathrm{m}$ and $18 \mu \mathrm{m}$ bands during the liquid helium period in the pointing mode data. 